\title{
Higgsed network calculus
}

\author{
Yegor Zenkevich ${ }^{1}$ \\ Dipartimento di Fisica, Università di Milano Bicocca, \\ Piazza della Scienza 3, I-20126 Milano, Italy \\ INFN, sezione di Milano-Bicocca, \\ I-20126 Milano, Italy \\ Institute for Theoretical and Experimental Physics (ITEP), \\ Moscow 117218, Russia \\ E-mail: yegor.zenkevich@gmail.com
}

ABSTRACT: We introduce a formalism for describing holomorphic blocks of $3 d$ quiver gauge theories using networks of Ding-Iohara-Miki algebra intertwiners. Our approach is very direct and gives an explicit identification of the blocks with Dotsenko-Fateev type integrals for $q$-deformed quiver $W$-algebras. We also explain how quiver theories corresponding to Dynkin diagrams of superalgebras arise, write down the corresponding partition functions and $W$-algebras, and explain the connection with supersymmetric Macdonald-Ruijsenaars commuting Hamiltonians.

KeYwords: Conformal and W Symmetry, Supersymmetric Gauge Theory, Topological Strings

ArXiv EPrint: 1812.11961

\footnotetext{
${ }^{1}$ Current affiliations:

SISSA, via Bonomea 265, 34136 Trieste, Italy,

INFN, Sezione di Trieste, IGAP, via Beirut 2/1, 34151 Trieste, Italy,

ITEP, Bolshaya Cheremushkinskaya street 25, 117218 Moscow, Russia,

ITMP MSU, Leninskie gory 1, 119991 Moscow, Russia.
} 


\section{Contents}

$\begin{array}{llr}1 & \text { Introduction } & 1\end{array}$

2 Intertwiners and blocks $\quad 4$

2.1 The intertwiners $\Phi$ and $\Phi^{*}$

2.2 Commutation relations for the intertwiners 8

2.3 Vertical gluing and $q$-Virasoro screening charges 9

$\begin{array}{lll}2.4 & q W_{N} \text {-algebra screenings and } 3 d \text { quivers } & 13\end{array}$

3 Bosonic and fermionic screenings $\quad 14$

$\begin{array}{lll}3.1 & \text { Dual screenings } & 14\end{array}$

$\begin{array}{ll}3.2 & \text { Gluing different Fock spaces. Fermionic screenings } \\ \end{array}$

4 Ruijsenaars Hamiltonians and their supersymmetric versions $\quad 19$

$\begin{array}{lll}5 & \text { Conclusions and discussions } & 21\end{array}$

A DIM algebra and its representations $\quad 22$

$\begin{array}{lll}\text { A.1 The algebra } & 22\end{array}$

$\begin{array}{lll}\text { A.2 The coproduct } & 23\end{array}$

A.3 Horizontal Fock representation 23

A.4 Vertical vector representation 24

$\begin{array}{lll}\text { A.5 Visualizing representations } & 25\end{array}$

\section{Introduction}

Ding-Iohara-Miki (DIM) algebra [1,2] is a unique and beautiful object. It can be understood as a quantum affinization $U_{q}(\widehat{\mathfrak{g}})$ of an algebra $\mathfrak{g}$ which is itself and affine algebra ${ }^{1}$ $\mathfrak{g}=\widehat{\mathfrak{g l}}_{1}$, deformed by an additional parameter $t$. Because of the presence of two loops in the construction, the algebra is often called quantum toroidal, and we will denote it by $U_{q, t}\left(\widehat{\hat{\mathfrak{g l}}}_{1}\right)$. DIM algebra is symmetric under the exchange of three parameters $q, t^{-1}$ and $\frac{t}{q}$. It has two gradings $\left(d, d_{\perp}\right)$, two central charges $\left(\gamma, \gamma_{\perp}\right)$ (again coming from two loops in the construction) and also an interesting automorphism group $\mathrm{SL}(2, \mathbb{Z})$ which acts on them as doublets. We collect the relevant definitions related to DIM algebra and its representations in appendix A.

In addition to being interesting from purely algebraic and representation theoretic point of view, DIM algebra is extremely relevant for physics. For example:

\footnotetext{
${ }^{1}$ The construction also works for $\mathfrak{g}=\widehat{\mathfrak{g l}}_{n}$.
} 
- It is the symmetry behind the AGT relation [3-5] between instanton series of $4 d$ $\mathcal{N}=2$ (and $5 d \mathcal{N}=1$ ) gauge theories and $2 d$ CFTs [6-8]. It is also the origin of the spectral duality, which exchanges the gauge groups at the nodes of a quiver gauge theory with the group corresponding to the Dynkin diagram of the quiver [9-13].

- It plays the central role in refined topological strings [14], where the central object of the formalism - refined topological vertex - can be identified with the intertwining operator of Fock representations of DIM algebra [6]. See eq. (2.1) for an illustration of such a vertex/intertwiner. It also endows toric Calabi-Yau three-folds with an interesting integrable structure $[15,16]$ and implies $(q, t)-\mathrm{KZ}$ difference equations for refined topological string amplitudes [18, 19].

- DIM algebra provides a universal way to understand "non-perturbative Ward identities", or $q q$-characters [20-34] for $4 d, 5 d$ and $6 d$ quiver gauge theories. Composing the trivalent intertwiners of Fock representations of DIM algebra according to a toric diagram of a CY threefold, one can build a "two-dimensional" (or network) matrix model, which is related to a family of Dotsenko-Fateev-like integral ensembles and the corresponding $q$-deformed vertex operator algebras [35].

- Very recently it was identified as the cohomological Hall algebra associated to CY three-folds [36-39].

- Higgsing construction can be employed to get holomorphic blocks [40,41] of $3 d$ $\mathcal{N}=2^{*}$ gauge theories ${ }^{2}$ from a specifically tuned network of intertwiners of DIM algebra $[42,43]$.

In the current paper we focus on the last item in the above list (however, as we will see there are further ramifications) and introduce a convenient formalism for describing $3 d$ $\mathcal{N}=2^{*}$ quiver gauge theories.

Let us first recall the Higgsing construction (for details see [42, 43]). The $3 d$ theories can be understood as worldvolume theories on the vortices in the Higgs phase of $5 d \mathcal{N}=1$ gauge theories [44-48]. In the $\Omega$-background there is an analogue of geometric transition $[49,50]$, which relates the theory with $M$ vortices in the Higgs phase to the theory without vortices in the Coulomb phase with scalar field vev $a=\epsilon_{2} M$. The geometric transition interpretation arises when we consider the Type IIB brane construction of the $5 d$ gauge theory. $M$ vortices on the Higgs branch correspond to $M$ D3 branes stretching between NS5 and D5' branes, while the dual side (after the transition) corresponds to the resolution of the NS5 and D5' crossing. The five-brane picture, in turn is related to the combination of DIM intertwiners, as shown in [6]. This combination gives the partition function of the Higgsed $5 d$ theory, and thus of the $3 d$ theory.

Our new formalism bypasses the complicated Higgsing procedure. The major source of complications in the Higgsing approach is the need to construct an auxiliary $5 d \mathcal{N}=1$

\footnotetext{
${ }^{2}$ By this we denote $\mathcal{N}=4$ gauge theories with supersymmetry softly broken by a real axial mass. For the details see $[42,43]$.
} 
a)

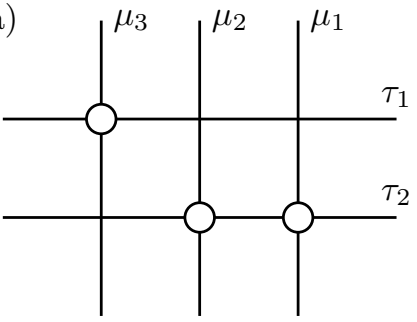

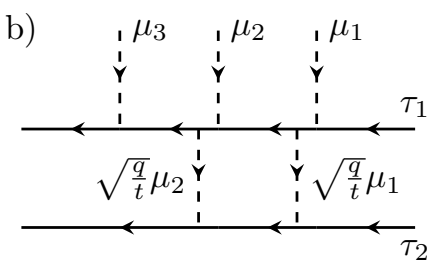

c)

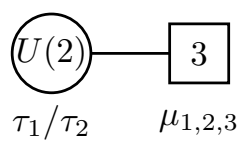

Figure 1. Comparison of two different formalisms for building a $3 d \mathcal{N}=2^{*}$ quiver gauge theory. a) The old formalism: horizontal and vertical solid lines denote horizontal and vertical Fock representations respectively; circles and crossings denote resolved conifold-like geometries with specially tuned parameters. The notation is explained in detail in [42, 43]. b) The new formalism. Solid horizontal lines still denote horizontal Fock spaces, while vertical dashed lines are vertical vector representations. They are joined together by Higgsed vertices. Notice how each circle in a) gives rise to dashed line emanating upwards from the corresponding point in b). Individual dashed lines correspond to screening charges acting on the Fock spaces represented by solid lines, so that the overall picture gives a Dotsenko-Fateev-like integral representation of the holomorphic block. c) The quiver gauge theory modelled by a) and b).

gauge theory, then tune its parameters to specific values, so that it reproduces the $3 d$ theory on the worldvolume of the vortex defects appearing in the Higgs branch of the $5 d$ theory.

Our new formalism, which we call the Higgsed network calculus, avoids the intermediate step (the auxiliary $5 d$ theory) and allows for direct computation of the holomorphic blocks of the $3 d$ theories. The formalism employs a "Higgsed" vertex which resembles refined topological vertex, but, unlike the latter, doesn't introduce a bend in the five-brane. An example of two descriptions of the same $3 d$ theory using the old and the new formalism is shown in figure 1. Overall, the Higgsed network looks as a collection of D3 branes (dashed lines in figure 1) stretched between a stack of parallel five-branes (solid lines).

On the algebraic side, Higgsed vertices can be thought of as elementary building blocks of screening currents, which commute with the action of a certain $W$-algebra and in this way can be used to define this algebra [51, 52]. It also turns out that in our approach we can easily reproduce the well-known result [53-56] — that the holomorphic blocks of $3 d$ gauge theories of the kind we are considering are eigenfunctions (or, more generally, kernel functions) of trigonometric Ruijsenaars-Schneider Hamiltonians.

Having thus reproduced the results of the old approach, we continue to some generalizations which are natural in the new formalism. We consider all three possible species of horizontal Fock representations and all three possible species of vertical vector representations and heavily employ the $\mathfrak{S}_{3}$ symmetry of the DIM algebra to build a network incorporating all of them (see appendix A for details on the representations of DIM algebra). This corresponds to introducing several sorts of screening currents. The resulting 
model was considered in [52], and corresponds to a $W$-algebra associated with a superalgebra. We have thus obtained partition functions of $3 d$ quiver theories corresponding to Dynkin diagrams of superalgebras. These theories should be $3 d$ uplifts of $2 d$ theories recently studied in [58] (see also earlier work [59]).

Indeed, the characteristic feature of the $2 d$ theories considered in [58] is that they are obtained by gluing together two linear quiver gauge theories of $A_{n-1}$ and $A_{m-1}$ type, corresponding to two bosonic subalgebras of the superalgebra $\mathfrak{g l}(n \mid m)$. Both parts are $\mathcal{N}=(4,4)$ theories softly broken to $\mathcal{N}=(2,2)$ by introducing the twisted mass for adjoint multiplets at each node of the quiver. The twisted masses of the adjoints in the two halves of the theory are opposite and the two halves are coupled through a certain $\mathcal{N}=(2,2)$ gauge theory forming the "fermionic" node of the quiver. As we will see, our construction naturally gives rise to partition functions which look very similar to this $2 d$ picture: they describe $3 d \mathcal{N}=2$ linear quiver gauge theories with adjoint matter multiplet on each gauge node and with adjoint mass having opposite signs on the two halves of the quiver which are coupled through a special "fermionic" node. We also prove that partition functions of the $3 d$ theories of these types are eigenfunctions of trigonometric Ruijsenaars-Schneider Hamiltonians or their supersymmetric generalizations [62].

The rest of the paper is structured as follows. In section 2 we present the systematic introduction of our formalism and examples of the computations reproducing the old construction: we write down one species of Higgsed vertices, show how to compose them into screening currents and derive the commutation relations for them. In section 3 we introduce the complete toolbox of vertices, build the fermionic screening current and write down the corresponding partition functions. We prove that the network partition functions are eigenfunctions of the supersymmetric Ruijsenaars-Schneider Hamiltonians in section 4. We present our conclusions and comment on future directions in section 5 .

\section{Intertwiners and blocks}

In this section we introduce the Higgsed vertices $\Phi$ and $\Phi^{*}$, from which we build the "Higgsed network". The vacuum matrix element of the network will give the partition function (holomorphic block) of the $3 d$ quiver gauge theory.

We have collected the definitions of the DIM algebra and its relevant representations in the appendix A not to clutter the presentation with too many technical details. However, the reader who is not familiar with DIM formalism is invited to consult it before proceeding to the main part of the text.

As a warm-up, let us recall the construction of the conventional refined topological vertices as intertwining operators of DIM algebra, proposed in [6]. In this approach fivebranes of Type IIB string theory (or, equivalently, the edges of the toric diagram of a CY threefold) are identified with Fock representations $\mathcal{F}_{u}^{(m, n), q, t^{-1}}$ of DIM algebra (see section A.3 for the definition of a Fock representation). The central charge vector of the Fock representation corresponds to the type of the fivebrane with (depending on an irrelevant choice of $\mathrm{SL}(2, \mathbb{Z})$ duality frame) $(1,0)$ meaning NS5 and $(0,1)$ meaning D5'. The 
intertwiner of Fock representation is a trivalent junction of branes, as shown below:

$$
\mathcal{F}_{-u w}^{(1,1), q, t^{-1}} \underbrace{\mathcal{F}_{w}^{(0,1), q, t^{-1}}}
$$

Since the charges of the representations, or branes are conserved, the branes are bend at the junction. The basis in a Fock space is labelled by Young diagrams. Therefore, solid lines in eq. (2.1) each carry a Young diagram, and gluing of two legs is performed by summing over a complete basis of states in the Fock space, i.e. over all Young diagrams. The result is just a network of intertwining operators, composed according to a five-brane web (or toric diagram of a CY). The vacuum matrix element of the network of intertwiners is equal to the refined topological string partition function on the toric CY [27, 28] (see also [29-34]).

\subsection{The intertwiners $\Phi$ and $\Phi^{*}$}

Let us introduce the main character of our story, the Higgsed vertex, or the vector intertwiner $\Phi(w): \mathcal{F}_{u}^{(1,0), q, t^{-1}} \otimes \mathcal{V}_{w}^{q} \rightarrow \mathcal{F}_{t u}^{(1,0), q, t^{-1}}$, which we draw as

$$
\Phi(w)=\mathcal{F}_{t u}^{(1,0), q, t^{-1}} \underbrace{\mathfrak{V}_{w}^{q}} \mathcal{F}_{u}^{(1,0), q, t^{-1}}
$$

From now on all the Fock representations we will encounter will have the "direction" of the central charge equal to $(1,0)$, and we omit it from our notation. The dashed vertical lines denote the vertical vector representations (see section A.4), while solid horizontal lines are still horizontal Fock representations (see section A.3), the latter exactly the same as in the ordinary DIM networks.

The vector representation has zero central charges, so when it joins a Fock representation the five-brane is not bent. This behavior reminds one of a D3 brane ending on an NS5 brane. In a moment we will see that this is not a coincidence and derive a precise relation between a network of intertwiners and the partition function of the effective $3 d$ theory obtained from the D3 branes stretched between a stack of five-branes.

The intertwining property of $\Phi$ means that $\Phi \Delta(g)=g \Phi$ for any element $g$ of DIM algebra (see the definition of the coproduct in section A.2). One can check that the following explicit expression ${ }^{3}[60,61]$ for the intertwiner $(2.2)$ indeed satisfies this constraint:

$$
\Phi(w)=e^{-\epsilon_{2} Q} w^{\frac{P}{\epsilon_{1}}} \exp \left[-\sum_{n \geq 1} \frac{w^{n}}{n} \frac{1-t^{-n}}{1-q^{n}} a_{-n}\right] \exp \left[\sum_{n \geq 1} \frac{w^{-n}}{n} \frac{1-t^{n}}{1-q^{-n}} a_{n}\right]
$$

where we define $q=e^{\epsilon_{1}}, t=e^{\beta \epsilon_{1}}=e^{-\epsilon_{2}}$ and the definition of the Fock space operators $a_{n}$, $P$ and $Q$ are given in eqs. (A.8), (A.9) in the appendix A. Notice that though the Fock space is not bent (its slope is horizontal all the way through the intertwiner), as we see from eq. (2.2) and the explicit expression (2.3), its spectral parameter (which plays the role

\footnotetext{
${ }^{3}$ We rescale the coordinate $w$ by $q^{-\frac{1}{2}}$ compared to $[60,61]$.
} 
of the position of the five-brane) is shifted after it passes the junction with the incoming dashed line.

We are already able to study the simplest nontrivial example, a network of two intertwiners:

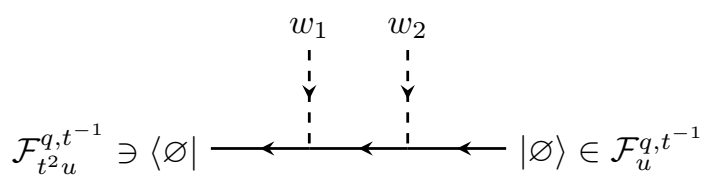

The corresponding expression is the matrix element of the product of two operators (2.3), which is evaluated by normal ordering the free fields:

$$
\begin{aligned}
(2.4)=\left\langle t^{2} u, \varnothing\left|\Phi\left(w_{1}\right) \Phi\left(w_{2}\right)\right| u, \varnothing\right\rangle & =w_{1}^{\log _{q} u+\beta} w_{2}^{\log _{q} u} \exp \left[-\sum_{n \geq 1} \frac{1}{n}\left(\frac{w_{2}}{w_{1}}\right)^{n} \frac{1-t^{-n}}{1-q^{-n}}\right]= \\
& =w_{1}^{\log _{q} u+\beta} w_{2}^{\log _{q} u} \frac{\left(\frac{q}{t} \frac{w_{2}}{w_{1}} ; q\right)_{\infty}}{\left(q \frac{w_{2}}{w_{1}} ; q\right)_{\infty}} .
\end{aligned}
$$

Moving one step further, we get an answer for the network of $n$ operators $\Phi$ glued together horizontally:

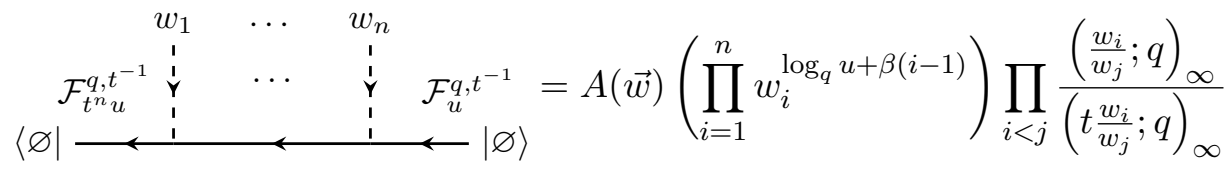

where we extract the $q$-periodic prefactor

$$
A(\vec{w})=\prod_{i<j}\left[\left(\frac{w_{i}}{w_{j}}\right)^{\beta} \frac{\theta_{q}\left(t \frac{w_{i}}{w_{j}}\right)}{\theta_{q}\left(\frac{w_{i}}{w_{j}}\right)}\right]
$$

from the matrix element; here $\theta_{q}(x)=(q ; q)_{\infty}(x ; q)_{\infty}\left(\frac{q}{x} ; q\right)_{\infty}$ is Jacobi theta-function. We will see in what follows that such $q$-periodic factors will be mostly immaterial to the structure of the network. In particular, they factor out of the sums when we glue vector states together, and give an overall $q$-periodic prefactor for the network.

To build networks of intertwiners similar to (but simpler than) those considered in $[6,27-34]$ we need one more operator, the dual intertwiner $\Phi^{*}(y): \mathcal{F}_{u}^{(1,0)} \rightarrow \mathcal{F}_{u / t}^{(1,0)} \otimes \mathcal{V}_{y}^{q}$, which satisfies $\Delta(g) \Phi^{*}=\Phi^{*} g$. An explicit check shows that the operator

$$
\begin{aligned}
& \underbrace{\mathcal{F}_{u}^{q, t^{-1}}}_{\substack{\mathcal{F}_{u t}^{q, t^{-1}} \\
\vdots \mathcal{V}_{y}^{q}}}=\Phi^{*}(y)= \\
& =e^{\epsilon_{2} Q} y^{\beta-\frac{P}{\epsilon_{1}}} \exp \left[\sum_{n \geq 1} \frac{y^{n}}{n}\left(\frac{t}{q}\right)^{\frac{n}{2}} \frac{1-t^{-n}}{1-q^{n}} a_{-n}\right] \exp \left[-\sum_{n \geq 1} \frac{y^{-n}}{n}\left(\frac{t}{q}\right)^{\frac{n}{2}} \frac{1-t^{n}}{1-q^{-n}} a_{n}\right]
\end{aligned}
$$

satisfies the intertwining property. Notice how the spectral parameter of the Fock space to the left of the dual intertwiner $\Phi^{*}$ is shifted the opposite way, compared to the intertwiner $\Phi$. 
The matrix elements of products of $\Phi^{*}$ are given by

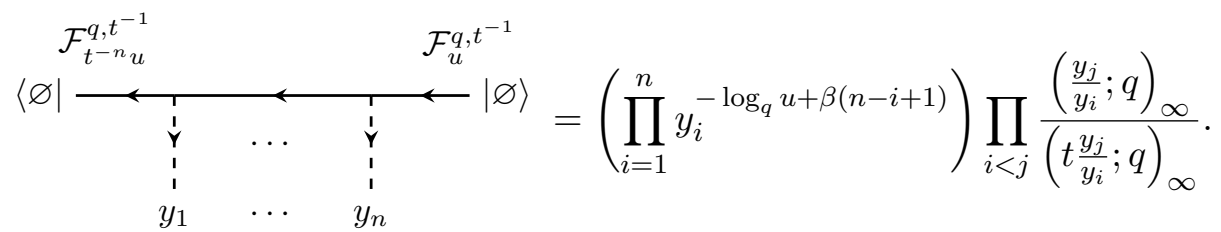

Combining $\Phi$ and $\Phi^{*}$ we get

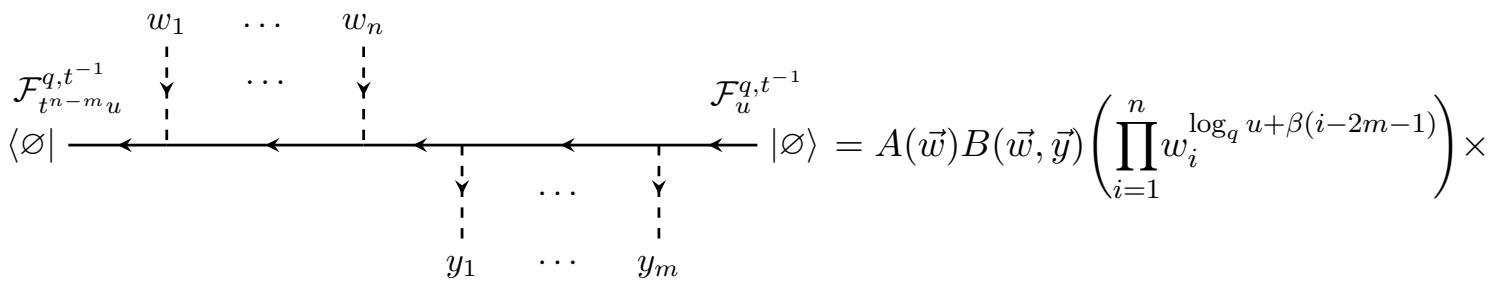

$$
\begin{aligned}
& \times\left(\prod_{i=1}^{m} y_{i}^{-\log _{q} u+\beta(i-2 n+1)}\right) \prod_{k<l}^{n} \frac{\left(\frac{w_{k}}{w_{l}} ; q\right)_{\infty}}{\left(t \frac{w_{k}}{w_{l}} ; q\right)_{\infty}} \prod_{i<j}^{m} \frac{\left(\frac{y_{j}}{y_{i}} ; q\right)_{\infty}}{\left(t \frac{y_{j}}{y_{i}} ; q\right)_{\infty}} \prod_{a=1}^{m} \prod_{b=1}^{n} \frac{\left(t \sqrt{\frac{q}{t}} \frac{w_{b}}{y_{a}} ; q\right)_{\infty}}{\left(\sqrt{\frac{q}{t}} \frac{w_{b}}{y_{a}} ; q\right)_{\infty}},
\end{aligned}
$$

where an additional $q$-periodic prefactor reads

$$
B(\vec{w}, \vec{y})=\prod_{a=1}^{m} \prod_{b=1}^{n}\left[\left(\frac{y_{a}}{w_{b}}\right)^{\beta} \frac{\theta_{q}\left(t \sqrt{\frac{q}{t}} \frac{y_{a}}{w_{b}}\right)}{\theta_{q}\left(\sqrt{\frac{q}{t}} \frac{y_{a}}{w_{b}}\right)}\right] .
$$

We can already notice that the $q$-Pochhammer factors in eq. (2.10) resemble those of the holomorphic block integrand for a pair of $\mathcal{N}=2$ bifundamental chiral multiplets charged under $\mathrm{U}(n) \times \mathrm{U}(m)$ flavour symmetry. In this case the parameter $q$ is identified with the parameter of the $3 d \Omega$-background $S^{2} \times{ }_{q} D_{2}$ and $t$ is related to the real axial mass deformation of the $\mathcal{N}=4$ theory. Together the two chirals constitute what we might call a bifundamental $\mathcal{N}=2^{*}$ multiplet, i.e. what remains of the $\mathcal{N}=4$ bifundamental multiplet after turning on the $t$-parameters responsible for the soft breaking of supersymmetry. Indeed, if we set the masses associated to $\mathrm{U}(n)$ flavours to $\mu_{i}=y_{i}, i=1, \ldots, n$ and the $\mathrm{U}(m)$ masses to $\bar{\mu}_{j}=\sqrt{\frac{q}{t}} w_{j}, j=1, \ldots, m$, we find that we reproduce the bifundamental contribution

$$
\prod_{a=1}^{m} \prod_{b=1}^{n} \frac{\left(t \frac{\bar{\mu}_{b}}{\mu_{a}} ; q\right)_{\infty}}{\left(\frac{\bar{\mu}_{b}}{\mu_{a}} ; q\right)_{\infty}}
$$

to the $D_{2} \times{ }_{q} S^{1}$ partition function. Thus, $n$ dashed lines coming from the top of the picture in eq. (2.10) correspond to a $\mathrm{U}(n)$ flavour group and $m$ dashed lines escaping from the bottom are related to $\mathrm{U}(m)$ flavour group. Thus, our initial guess that the dashed lines are somehow associated with D3 branes seem to be plausible: the $3 d$ bifundamental chirals couple the $\mathrm{U}(n)$ and $\mathrm{U}(m)$ gauge theories living on the stacks of D3 branes. The D3 branes are semi-infinite, so the gauge fields are frozen and the gauge symmetries become flavour symmetries of the $3 d$ theory of bifundamental chirals.

What is the field theory role of the remaining factors in eq. (2.10)? First of all, the $q$ periodic contributions in the holomorphic blocks are not important, since they cancel when 
one combines two blocks into a partition function for a compact manifold (e.g. $S_{b}^{3}$ ). Additional $q$-Pochhammers in eq. (2.10) can be thought of as coming from flipping fields [42, 43], charged under flavour symmetries $\mathrm{U}(n)$ and $\mathrm{U}(m)$.

We will see in what follows that when we glue pictures like (2.10) along the dashed lines we effectively gauge the corresponding flavour groups. The flipping fields coming from both sides then combine into an $\mathcal{N}=2$ vector and adjoint chiral contribution for the gauged symmetry. This is in accordance with the D3 brane interpretation: the couplings on the branes are unfrozen, when the branes have finite length.

\subsection{Commutation relations for the intertwiners}

For the moment we have only shown how to compose the intertwiners $\Phi$ and $\Phi^{*}$ horizontally. However, we can already make a natural and meaningful exercise with our building blocks. Let us compare different orderings of the intertwiners along the solid line. There are three possibilities:

1. Commutation of $\Phi$ with $\Phi$ :

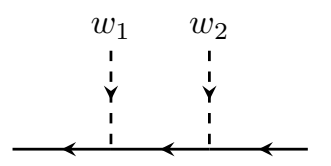

$$
=\left[\left(\frac{w_{1}}{w_{2}}\right)^{\beta} \frac{\theta_{q}\left(t \frac{w_{1}}{w_{2}}\right)}{\theta_{q}\left(\frac{w_{1}}{w_{2}}\right)}\right] R\left(\frac{w_{1}}{w_{2}}\right) \times
$$

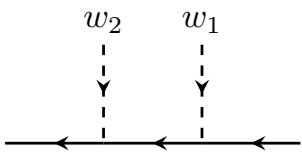

where

$$
R(x)=\frac{(x ; q)_{\infty}(q x ; q)_{\infty}}{(t x ; q)_{\infty}\left(\frac{q}{t} x ; q\right)_{\infty}} .
$$

The terms in the square brackets in eq. (2.13) combine into a $q$-periodic function of $w_{1,2}$, which, as we have mentioned above, is not important for our network construction. The function $R(x)$ is the "miniature version" of the DIM $R$-matrix [15, 16, 6871 . In our case the $R$-matrix permutes two vector representations living on the vertical dashed lines.

2. Commutation of $\Phi^{*}$ with $\Phi^{*}$. For the dual intertwiners we find

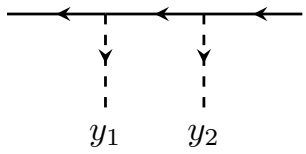

$$
=\left[\left(\frac{y_{1}}{y_{2}}\right)^{\beta} \frac{\theta_{q}\left(\frac{y_{2}}{y_{1}}\right)}{\theta_{q}\left(t \frac{y_{2}}{y_{1}}\right)}\right] \frac{1}{R\left(\frac{y_{1}}{y_{2}}\right)} \times
$$

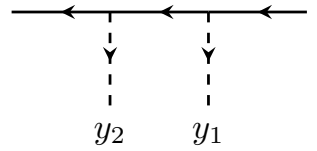

which features an inverse of the $R$-matrix from eq. (2.14) together with another $q$ periodic factor.

3. Commutation of $\Phi$ with $\Phi^{*}$. Finally, we have

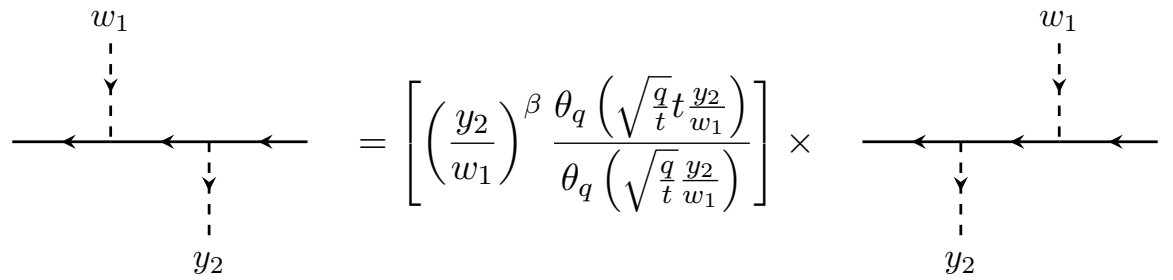

We find that $\Phi$ and $\Phi^{*}$ commute, up to $q$-periodic factors, which for us is as good as commutativity. 


\subsection{Vertical gluing and $q$-Virasoro screening charges}

We pass to the next necessary step in building the network of intertwiners - vertical gluing. In our convention the states in the vector representation correspond to the shifts of the spectral parameter $w \mapsto q^{k} w$. Thus, to sum over the complete basis of states in the vector representation we need to take the sum over $k$, or, equivalently, the so-called Jackson $q$-integral over the spectral parameter $w$ of the vector representation. For example, gluing together two dual intertwiners we get an operator $\mathcal{Q}_{q}^{q, t^{1}}: \mathcal{F}_{u_{1}}^{q, t^{-1}} \otimes \mathcal{F}_{u_{2}}^{q, t^{-1}} \rightarrow \mathcal{F}_{t^{-1} u_{1}}^{q, t^{-1}} \otimes \mathcal{F}_{t u_{2}}^{q, t^{-1}}$ :

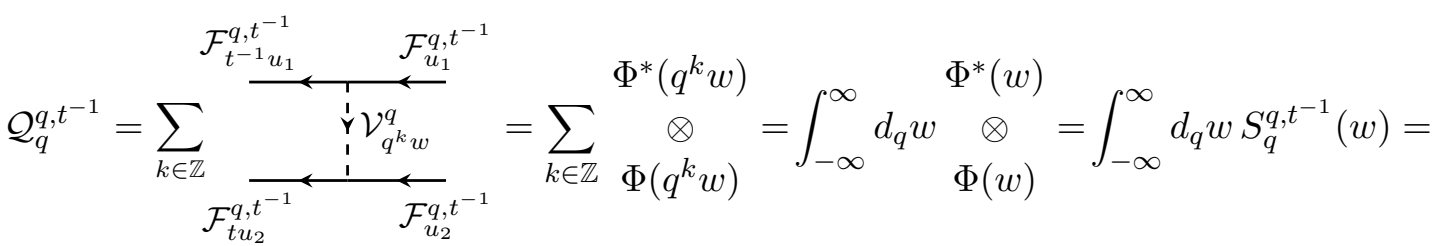

$$
\begin{aligned}
& =\sum_{k \in \mathbb{Z}} e^{-\epsilon_{2}\left(Q_{1}-Q_{2}\right)}\left(q^{k} w\right)^{\beta+\frac{P_{1}-P_{2}}{\epsilon_{1}}} \exp \left[-\sum_{n \geq 1} \frac{w^{n}}{n} q^{n k} \frac{1-t^{-n}}{1-q^{n}}\left(a_{-n}^{(2)}-\left(\frac{t}{q}\right)^{\frac{n}{2}} a_{-n}^{(1)}\right)\right] \times \\
& \times \exp \left[\sum_{n \geq 1} \frac{w^{-n}}{n} q^{-n k} \frac{1-t^{n}}{1-q^{-n}}\left(a_{n}^{(2)}-\left(\frac{t}{q}\right)^{\frac{n}{2}} a_{n}^{(1)}\right)\right],
\end{aligned}
$$

where we denote by $a_{n}^{(1)}$ (resp. $a_{n}^{(2)}$ ) creation and annihilation operators acting on the upper (resp. lower) horizontal Fock space (similarly for the zero modes $P_{1,2}$ and $Q_{1,2}$ ). One more convenient representation of the vertical gluing is the contour integral over an appropriate contour in the complex $w$ plane. One way to rewrite eq. (2.17) as a contour integral is to notice that

$$
\frac{(q ; q)_{\infty}^{2}}{(a ; q)_{\infty}\left(\frac{q}{a} ; q\right)_{\infty}} \oint_{\mathcal{C}} \frac{d \xi}{\xi}\left(\frac{\xi}{w}\right)^{\log _{q} a} \frac{\theta_{q}\left(\frac{a \xi}{w}\right)}{\theta_{q}\left(\frac{\xi}{w}\right)} f(\xi)=\sum_{k \in \mathbb{Z}} f\left(q^{k} w\right),
$$

for almost any $a \in \mathbb{C}$, where $\mathcal{C}$ wraps the poles of the theta-function in the denominator. Therefore, by inserting the theta-functions and a prefactor, as in eq. (2.18), under the integral and integrating over a specific contour we can turn the integral into a sum. It turns out that for certain combinations of intertwiners the contour $\mathcal{C}$ can be traded for other contours wrapping poles of the correlation functions (2.10). As have been noted in e.g. [8, 13], eventually all the representations of the "trigonometric integrals" — either as a sum, or as a Jackson $q$-integral, or as a contour integral - boil down to the same expression and are completely equivalent. Similar equivalence occurs in the DotsenkoFateev representations of $q$-deformed conformal blocks and in the holomorphic blocks of $3 d$ theories. We will mostly use the contour integral representation, usually assuming that the contour wraps the poles of the correlation functions under the integral.

In fact, the integrand $S_{q}^{q, t^{-1}}(w)$ in the first line of eq. (2.17) is nothing but the screening current of the $q$-Virasoro algebra $\operatorname{Vir}_{q, t}$, built from the pair of free bosons $a_{n}^{(1,2)}[27,28]$. Thus $\mathcal{Q}_{q}^{q, t^{-1}}$ is the screening charge, commuting with the action of $\operatorname{Vir}_{q, t}$. As explained in $[27,28,63-67]$ this algebra is a subalgebra of the DIM algebra, i.e. one can build a current from a combination of DIM generators, so that when acting on the tensor product of two 
horizontal Fock representations it reproduces the relations of $\mathrm{Vir}_{q, t}$. Since the network of intertwiners (2.17) commutes with $\Delta(g)$ for any $g$ from the DIM algebra, it also commutes with the $q$-Virasoro current. We can write the intertwining relation graphically:

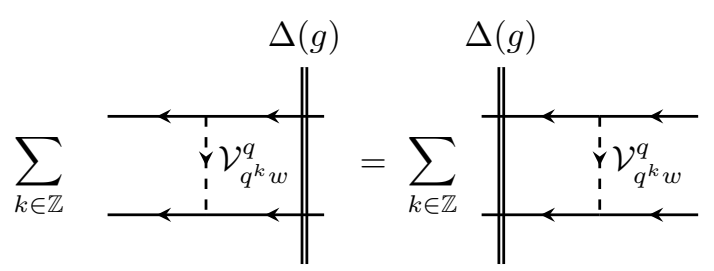

where the double line denotes the position of the operator $\Delta(g)$ on the horizontal lines, or as a formula

$$
\left[\mathcal{Q}_{q}^{q, t^{-1}}, \Delta(g)\right]=0 .
$$

A more general network can be obtained by adding external dashed lines, e.g.

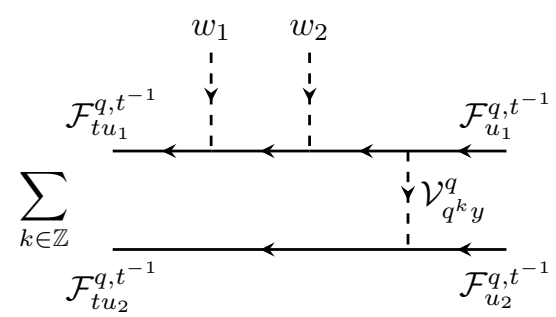

One can see that the external lines correspond to degenerate vertex operators of the $\mathrm{Vir}_{q, t}$ algebra, similarly to $[27,28]$. The network $(2.21)$ is therefore a product of screened degenerate vertex operators. Taking the vacuum matrix element of eq. (2.21) we get

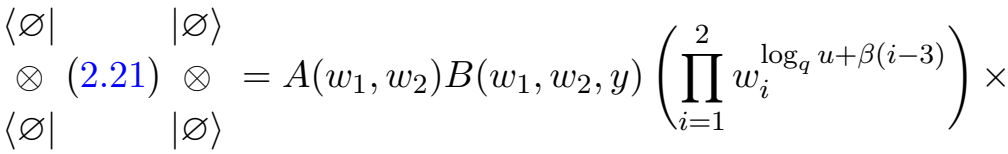

$$
\begin{aligned}
& \times \frac{\left(\frac{w_{1}}{w_{2}} ; q\right)_{\infty}}{\left(t \frac{w_{1}}{w_{2}} ; q\right)_{\infty}} \int_{-\infty}^{\infty} d_{q} y y^{\log _{q} \frac{u_{2}}{u_{1}}-2 \beta} \prod_{i=1}^{2} \frac{\left(t \sqrt{\frac{q}{t}} \frac{w_{i}}{y} ; q\right)_{\infty}}{\left(\sqrt{\frac{q}{t}} \frac{w_{i}}{y} ; q\right)_{\infty}}
\end{aligned}
$$

where $A(\vec{w})$ and $B(\vec{w}, \vec{y})$ are periodic factors from eq. (2.7) and from eq. (2.11) respectively.

The $3 d$ gauge theory corresponding to the network (2.21) can be deduced either from the form of the integrand (2.22) ( $q$-Pochhammer ratios give two pairs of chirals and a single integration implies a $\mathrm{U}(1)$ gauge group) or directly by interpreting (2.21) as a brane picture (the intermediate dashed line gives the $\mathrm{U}(1)$ gauge theory and two upper dashed lines contribute two fundamental multiplets, i.e. two pairs of fundamental chirals). In any case, the resulting theory is the $\mathcal{N}=2^{*}$ version of the $T[\mathrm{SU}(2)]$ theory. The masses of the fundamental multiplets are $\mu_{i}=\sqrt{\frac{q}{t}} w_{i}$ and the FI parameter of the U(1) gauge group is $\tau=\frac{u_{2}}{u_{1}}$. The prefactor in the second line of eq. (2.22) corresponds to flipping fields of the $\mathrm{U}(2)$ flavour symmetry group.

Let us make a comment about the contours, over which one can integrate the screening currents. If we consider the space of intertwining operators of the form (2.21) as a vector space over $q$-periodic functions of the parameters, it turns out to be two dimensional. One can obtain this fact using several different lines of arguments: 
1. As we show in section 4 the network (2.21) is an eigenfunctions of the Ruijsenaars difference operator with the eigenvalue independent of $y$. $q$-periodic functions pass through the difference operator, so one can study the space of eigenfunctions as a vector space over the field of $q$-periodic functions in the same way as one studies the space of eigenfunctions of a differential operator over the field of constants. The difference operator is of second order, therefore the space of solutions is two-dimensional.

2. The $3 d$ theory $T[\mathrm{SU}(2)]$ corresponding to the network has two vacua. These vacua correspond to two linearly independent networks of intertwiners.

We can write two linearly independent networks of intertwiners similarly to (2.22), but with the Jackson $q$-integrals replaced by the contour integrals over the contours $\mathcal{C}_{1,0}$ and $\mathcal{C}_{0,1}$, wrapping the poles at $y=\sqrt{\frac{q}{t}} w_{1} q^{k}, k \in \mathbb{Z}_{\geq 0}$ and $y=\sqrt{\frac{q}{t}} w_{2} q^{k}, k \in \mathbb{Z}_{\geq 0}$ respectively. One can also isolate the contributions of contours $\mathcal{C}_{1,0}$ and $\mathcal{C}_{0,1}$ by taking the residue in the $y$-parameter of the intermediate dashed line at $y=\sqrt{\frac{q}{t}} w_{1,2}$.

In what follows, not to overburden the notation, we will omit writing the sums and the shifts of the intermediate dashed lines on our diagrams. In this convention two networks corresponding to contours $\mathcal{C}_{1,0}$ and $\mathcal{C}_{0,1}$ are given by the following pictures
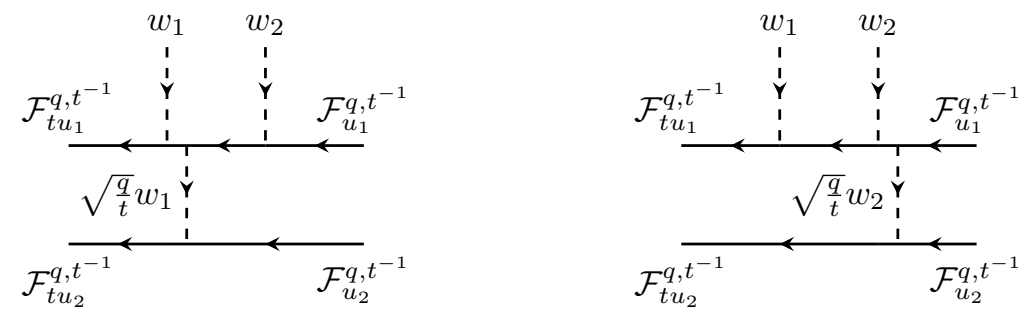

Notice how the lower dashed lines "cling" to the upper dashed lines. As we have explained, this is the consequence of the structure of poles of the integrand. For several screenings stretched between two neighbouring horizontal lines (i.e. for a non-abelian $3 d$ theory), from the first look at the structure of the integrand one could have assumed that the intermediate lines can also "cling" together forming stacks of branes. Indeed, the interaction between two parallel branes gives a denominator $\left(t \frac{y_{k}}{y_{l}} ; q\right)_{\infty}$. For example, the picture:

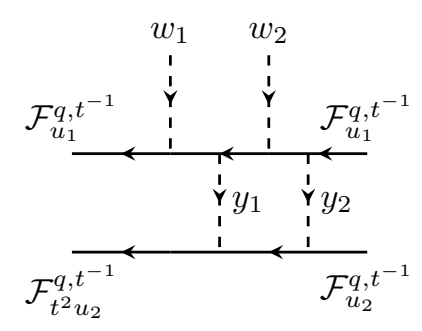

corresponds to the integral

$$
\oint_{\mathcal{C}} \frac{d^{2} y}{y_{1} y_{2}}\left(y_{1} y_{2}\right)^{\log _{q} \frac{u_{2}}{u_{1}}-2 \beta} \prod_{k \neq l}^{2} \frac{\left(\frac{y_{k}}{y_{l}} ; q\right)_{\infty}}{\left(t \frac{y_{k}}{y_{l}} ; q\right)_{\infty}} \prod_{i=1}^{2} \prod_{j=1}^{2} \frac{\left(t \sqrt{\frac{q}{t}} \frac{w_{i}}{y_{j}} ; q\right)_{\infty}}{\left(\sqrt{\frac{q}{t}} \frac{w_{i}}{y_{j}} ; q\right)_{\infty}}
$$


This "stacking" of branes would have corresponded to the contour of double integration $\mathcal{C}=\mathcal{C}_{0,2}$ over the spectral parameters $y_{1}$ and $y_{2}$, which wraps the poles $y_{1}=\sqrt{\frac{q}{t}} w_{2} q^{k_{1}}$, $y_{2}=\sqrt{\frac{q}{t}} w_{2} q^{k_{2}} t^{-1}$, with $k_{1,2} \in \mathbb{Z}_{\geq 0}$. However, in fact this group of poles gets cancelled by the numerators $\left(t \sqrt{\frac{q}{t}} \frac{w_{i}}{y_{j}} ; q\right)_{\infty}$ in the integrand (2.25). Therefore parallel dashed lines between the same horizontal lines cannot cling together and always cling to separate upper dashed lines.

As one can deduce from the brane diagram, the $3 d$ theory corresponding to the network (2.24) is the $\mathrm{U}(2)$ gauge theory (two intermediate dashed lines) with two fundamental multiplets (two external dashed lines). It has only one arrangement of branes in which $y_{1,2}=\sqrt{\frac{q}{t}} w_{1,2} q^{k_{1,2}}$. Thus, the resulting theory has only one vacuum. Geometrically, this theory corresponds to counting certain quasimaps to the target $\operatorname{Gr}(2,2) \simeq \operatorname{Gr}(0,2)$, i.e. to a point.

In general, the network with two horizontal lines corresponds to a $3 d$ with a single gauge group $\mathrm{U}(m)$ and a number $n$ of fundamental multiplets:

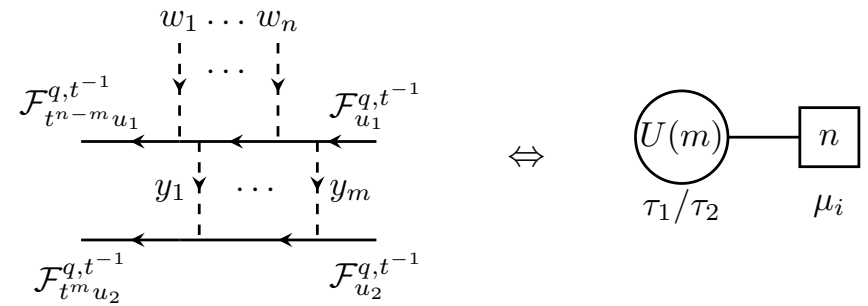

There are $\frac{n !}{m !(n-m) !}$ vacua, corresponding to the contours of integration $\mathcal{C}_{1,0, \ldots, 1}$ with $m$ ones and $(n-m)$ zeroes. Graphically this number is explained as follows: $m$ intermediate dashed lines can cling to separate external dashed line, and all the intermediate dashed lines are identical, hence $m$ ! in the denominator. These vacua are identified with fixed points of $\left(\mathbb{C}^{*}\right)^{n}$ action on $\operatorname{Gr}(m, n)$ (notice that we consider only $m \leq n$ ). The corresponding integrals are given by

$$
\underset{\langle\varnothing|}{\otimes(2.26) \underset{|\varnothing|}{|\varnothing\rangle} \otimes} \sim \prod_{k<l}^{n} \frac{\left(\frac{w_{k}}{w_{l}} ; q\right)_{\infty}}{\left(t \frac{w_{k}}{w_{l}} ; q\right)_{\infty}} \oint_{\mathcal{C}_{k_{1}, \ldots, k_{n}}} d^{m} y \prod_{i=1}^{m} y^{\log _{q} \frac{u_{2}}{u_{1}}-2 \beta-1} \frac{\Delta_{m}^{(q, t)}(\vec{y})}{\bar{\Delta}_{m, n}^{(q, t)}(\vec{y}, \vec{w})},
$$

where

$$
\Delta_{m}^{(q, t)}(\vec{y})=\prod_{k \neq l}^{m} \frac{\left(\frac{y_{k}}{y_{l}} ; q\right)_{\infty}}{\left(t \frac{y_{k}}{y_{l}} ; q\right)_{\infty}}, \quad \bar{\Delta}_{m, n}^{(q, t)}(\vec{y}, \vec{w})=\prod_{i=1}^{n} \prod_{j=1}^{m} \frac{\left(\sqrt{\frac{q}{t}} \frac{w_{i}}{y_{j}} ; q\right)_{\infty}}{\left(t \sqrt{\frac{q}{t}} \frac{w_{i}}{y_{j}} ; q\right)_{\infty}}
$$

This is precisely the integral for the holomorphic block of the $\mathrm{U}(m)$ theory with $n$ fundamental multiplets. The prefactors again play the role of flipping fields of the flavour symmetry. We can relate the parameters of the network to that of the gauge theory on $S^{1} \times{ }_{q} \mathbb{R}^{2}$. We write down the dictionary in table 1 . 


\begin{tabular}{|c|c|}
\hline $3 d \mathrm{U}(m)$ gauge theory & Higgsed network $(2.21)$ \\
\hline Axial real mass $m_{A}$ & $\frac{1}{R_{S 1}} \ln \left(-\frac{\sqrt{q}}{t}\right)$ \\
$3 d \Omega$-background parameter & $q$ \\
FI parameter $\tau$ & $\frac{u_{2}}{u_{1}}$ \\
Flavour masses $\mu_{i}$ & $\sqrt{\frac{q}{t}} w_{i}$ \\
Vacua & Contours of integration \\
\hline
\end{tabular}

Table 1. Dictionary between the parameters of the $3 d$ gauge theory and Higgsed network.

\section{4 $q W_{N}$-algebra screenings and $3 d$ quivers}

We can stack more than two horizontal Fock representations on top of each other and stretch vector representations between them. For example:

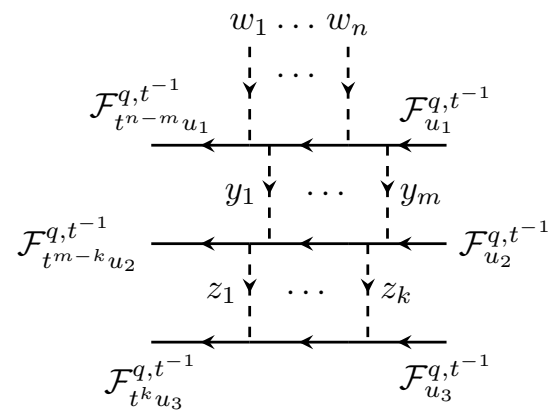

The intermediate dashed lines can now stretch between either the upper two Fock spaces or the lower two. This gives rise to two screening currents, $S_{q, 12}^{q, t^{-1}}(y)$ and $S_{q, 23}^{q, t^{-1}}(z)$ (and the corresponding screening charges), which are similar to the Virasoro one (2.17) with appropriate change of $a_{n}^{(1,2)}$ to $a_{n}^{(2,3)}$ in $S_{q, 23}^{q, t^{-1}}(z)$. The algebra commuting with the screening charges is the $q$-deformed $W_{3}$-algebra. The commutation can be inferred from the DIM intertwining relations exactly as in eq. (2.19).

Two sorts of screening currents have nontrivial normal ordering, because the bosonic operators $a_{n}^{(2)}$ are shared between them. In fact the normal ordering produces the interaction, dictated by the $A_{2}$ Cartan matrix, between the integration variables $y_{i}$ and $z_{j}$. Thus, the vacuum matrix element of the network (2.29) is the $A_{2}$-type $q$-conformal matrix model, as in [13]:

$$
\begin{aligned}
& \langle\varnothing|\quad| \varnothing\rangle \\
& \underset{\otimes \varnothing|(2.29)| \varnothing\rangle}{\otimes} \sim \prod_{k<l}^{n} \frac{\left(\frac{w_{k}}{w_{l}} ; q\right)_{\infty}}{\left(t \frac{w_{k}}{w_{l}} ; q\right)_{\infty}} \times \\
& \langle\varnothing|\quad| \varnothing\rangle \\
& \times \oint_{\mathcal{C}_{k_{1}, \ldots, k_{n}}} d^{m} y \oint_{\mathcal{C}_{l_{1}, \ldots, l_{k}}} d^{k} z \prod_{i=1}^{m} y^{\log _{q} \frac{u_{2}}{u_{1}}-2 \beta-1} \prod_{i=1}^{k} z^{\log _{q} \frac{u_{3}}{u_{2}}-2 \beta-1} \frac{\Delta_{k}^{(q, t)}(\vec{z}) \Delta_{m}^{(q, t)}(\vec{y})}{\bar{\Delta}_{k, m}^{(q, t)}(\vec{z}, \vec{y}) \bar{\Delta}_{m, n}^{(q, t)}(\vec{y}, \vec{w})},
\end{aligned}
$$


where $\Delta$ and $\bar{\Delta}$ are given in eq. (2.28). The corresponding gauge theory is a linear quiver

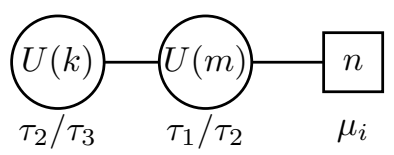

For $N$ horizontal lines there will be $(N-1)$ intermediate stacks of D3 branes and correspondingly, the $3 d$ quiver would have $(N-1)$ nodes, since each D3 stack corresponds to a gauge group.

\section{Bosonic and fermionic screenings}

In this section we follow the natural development of our formalism and introduce additional intertwiners, which are obtained by acting with the $\mathfrak{S}_{3}$ permutation symmetry of DIM on $\Phi$ and $\Phi^{*}$. When combined into a network, these new intertwiners produce new screening currents and charges, defining more general $W$-algebras and $3 d$ quiver gauge theories, which both turn out to be associated with superalgebras. ${ }^{4}$ In this way we reproduce the results of [52] on $W$-algebras associated to DIM algebra in a simplified and streamlined way. More concretely, our intertwiners are "halfs" of the screening currents which appeared in [52], i.e. a pair of intertwiners contracted together gives a screening charge. Thus the new intertwiners can be thought of as elementary building blocks, from which the screening charges of [52] can be constructed.

\subsection{Dual screenings}

As shown in section A.3, the Fock space representation $\mathcal{F}_{u}^{q, t^{-1}}$ of DIM is invariant under the symmetry $q \leftrightarrow t^{-1}$. However, the intertwining operators, $\Phi$ and $\Phi^{*}$ from eqs. (2.3) and (2.8) respectively are not invariant. This is not a surprise of course, since the operators $\Phi$ and $\Phi^{*}$ intertwine tensor products of $\mathcal{F}_{u}^{q, t^{-1}}$ with $V_{q}^{w}$ and while the former is invariant under $q \leftrightarrow t^{-1}$, the latter is not. Acting with the symmetry $q \leftrightarrow t^{-1}$ on $\Phi$ and $\Phi^{*}$ we obtain new intertwiners. At this point we need to refine our notation slightly in order not to confuse different operators. We call the intertwiners $\Phi$ and $\Phi^{*}$ from eqs. (2.3) and (2.8) $\Phi_{q, t^{-1}}^{q}(w)$ and $\Phi_{q}^{* q, t^{-1}}(w)$ with the indices signifying the spaces they act on. We also introduce the color-coded graphical notation with three vector representations $\mathcal{V}_{w}^{q}, \mathcal{V}_{w}^{t^{-1}}$ and $\mathcal{V}_{w}^{t / q}$ drawn as blue, red and violet dashed lines respectively. In this way each parameter $\left(q, t^{-1}, t / q\right)$ corresponds to a color: $q$ to blue, $t^{-1}$ to red, $\frac{t}{q}$ to violet. The Fock spaces also carry the color corresponding to their "missing index": $\mathcal{F}_{u}^{q, t^{-1}}$ is violet, $\mathcal{F}_{u}^{q, t / q}$ is red, $\mathcal{F}_{u}^{t^{-1}, t / q}$ is blue.

\footnotetext{
${ }^{4} \mathrm{An}$ important distinction should be made between gauge theories with gauge supergroups and quiver gauge theories with conventional bosonic gauge groups but with quivers having the form of Dynkin diagrams associated with classical Lie superalgebras. Here by the "association" between the gauge theory and superalgebra we mean the latter.
} 
The intertwiners $\Phi_{q, t^{-1}}^{q}(w)$ and $\Phi_{q}^{* q, t^{-1}}(w)$ are then drawn as

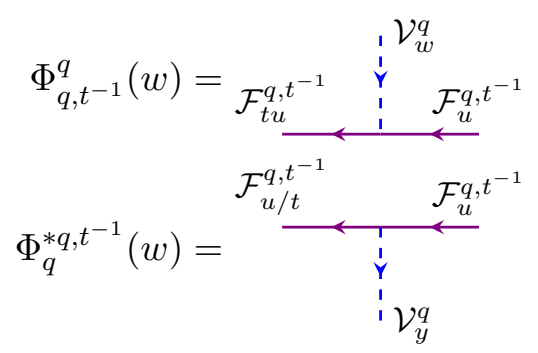

The new intertwiners are drawn as

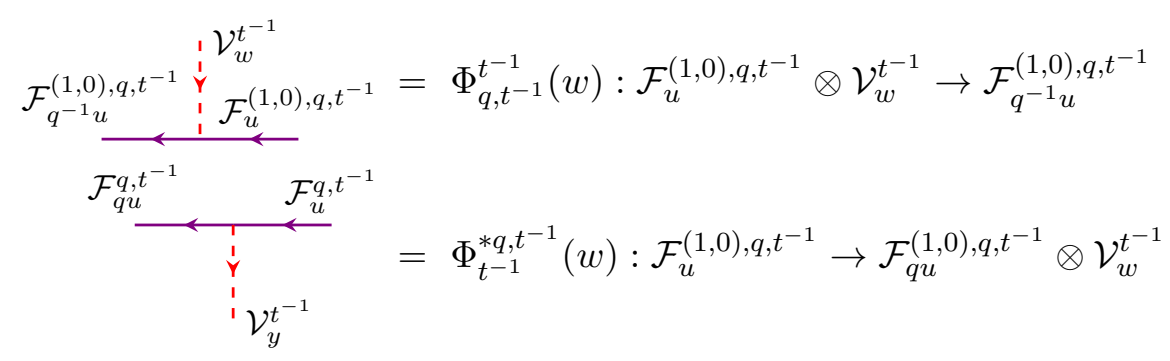

The explicit expressions for the new intertwiners are as follows:

$$
\begin{aligned}
\Phi_{q, t^{-1}}^{t^{-1}}(w) & =e^{-\epsilon_{1} Q} w^{\frac{P}{\epsilon_{2}}} \exp \left[-\sum_{n \geq 1} \frac{w^{n}}{n} a_{-n}\right] \exp \left[\sum_{n \geq 1} \frac{w^{-n}}{n} a_{n}\right], \\
\Phi_{t^{-1}}^{* q, t^{-1}}(w) & =e^{\epsilon_{1} Q} y^{\frac{1}{\beta}-\frac{P}{\epsilon_{2}}} \exp \left[\sum_{n \geq 1} \frac{y^{n}}{n}\left(\frac{t}{q}\right)^{\frac{n}{2}} a_{-n}\right] \exp \left[-\sum_{n \geq 1} \frac{y^{-n}}{n}\left(\frac{t}{q}\right)^{\frac{n}{2}} a_{n}\right],
\end{aligned}
$$

where we have applied the symmetry (A.14) to the intertwiners (2.3) and (2.8). Of course, all the results for normal ordering, commutation and gluing obtained in section 2 hold for the red dashed lines as well, provided one exchanges $q \leftrightarrow t^{-1}$ in all the expressions (hence, for example, the integrations over the spectral parameters of the intermediate red dashed lines are $t^{-1}$-Jackson integrals and the irrelevant prefactors are $t$-periodic).

One can use the new intertwiners together with the "old" ones to obtain more general networks. The color rule for gluing the colored lines is simple: the intertwiners we have just described (eqs. (3.1)-(3.4)) connect dashed lines and solid lines of colors which do not coincide. For example, a red dashed line can connect to blue and violet, but not red horizontal lines. Let us for the moment consider horizontal lines of only one color, say violet (the general setup will be described in section 3.2).

For the simplest example, consider two violet horizontal lines. We can stretch either blue or red dashed lines between them:

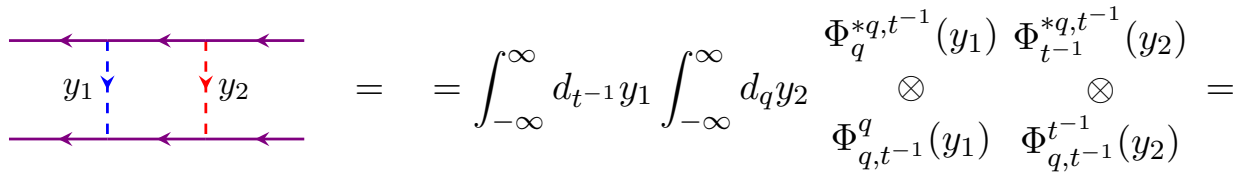

$$
\begin{aligned}
& =\int_{-\infty}^{\infty} d_{t^{-1}} y_{1} \int_{-\infty}^{\infty} d_{q} y_{2} S_{q}^{q, t^{-1}}\left(y_{1}\right) S_{t^{-1}}^{q, t^{-1}}\left(y_{2}\right) \text {. }
\end{aligned}
$$


These two types of lines give rise to two types of screening currents $S_{q}^{q, t^{-1}}(w)$ ("blue" current) and $S_{t^{-1}}^{q, t^{-1}}(w)$ ("red" current). Both of them commute with the action of DIM algebra, and therefore with the action of the $q$-Virasoro acting on the Fock spaces. In fact these two screenings also commute with each other and constitute the well-known standard set of screenings of the $q$-Virasoro algebra.

To give a more familiar example of the same situation consider the ordinary Virasoro algebra built from a free field $\phi(x)$ and generated by $T(z)=(\partial \phi(z))^{2}+\frac{b-b^{-1}}{2} \partial^{2} \phi(z)$. Then there are two stanrard screening currents $: e^{b \phi(x)}:$ and $: e^{\frac{1}{b} \phi(x)}:$ commuting with $T(z)$ related by the symmetry $b \leftrightarrow \frac{1}{b}$. This symmetry is exactly the symmetry $q \leftrightarrow t^{-1}$ of the $q$-deformed model.

As noted in [52], the red and blue screening currents commute:

$$
\left[S_{q}^{q, t^{-1}}(w), S_{t^{-1}}^{q, t^{-1}}(y)\right]=0 .
$$

However, their normal ordering is nontrivial, though it doesn't contain $q$-Pochhammer symbols as the ordering between screening currents of the same color:

$$
S_{t^{-1}}^{q, t^{-1}}\left(y_{1}\right) S_{q}^{q, t^{-1}}\left(y_{2}\right)=\frac{y_{1}^{2}}{\left(1-\frac{1}{t} \frac{y_{2}}{y_{1}}\right)\left(1-\frac{1}{q} \frac{y_{2}}{y_{1}}\right)}: S_{t^{-1}}^{q, t^{-1}}\left(y_{1}\right) S_{q}^{q, t^{-1}}\left(y_{2}\right):
$$

Thus, the integrals corresponding to the networks with two violet horizontal lines can be described as a pair of coupled $q$-Dotsenko-Fateev-type integral ensembles:

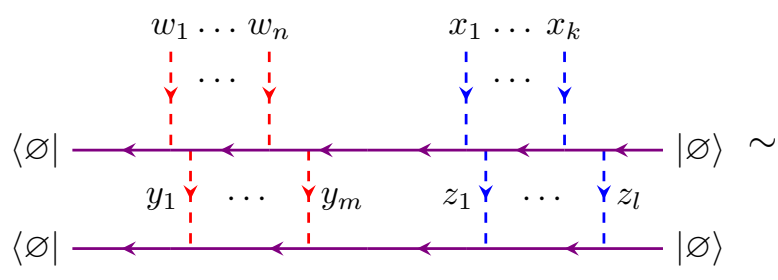

$$
\begin{aligned}
& \sim \oint_{\mathcal{C}_{k_{1}, \ldots, k_{n}}} d^{m} y \oint_{\mathcal{C}_{l_{1}, \ldots, l_{k}}} d^{l} z \prod_{i=1}^{m} y^{-\log _{t} \frac{u_{2}}{u_{1}}-\frac{2}{\beta}-1} \frac{\Delta_{m}^{\left(t^{-1}, q^{-1}\right)}(\vec{y})}{\bar{\Delta}_{m, n}^{\left(t^{-1}, q^{-1}\right)}(\vec{y}, \vec{w})} \prod_{i=1}^{l} z^{\log _{\frac{1}{u_{1}}} u_{2}-2 \beta-1} \frac{\Delta_{l}^{(q, t)}(\vec{z})}{\bar{\Delta}_{l, k}^{(q, t)}(\vec{z}, \vec{x})} \\
& \prod_{i=1}^{l} \prod_{j=1}^{m} \frac{1}{\left(z_{i}-\frac{1}{t} y_{j}\right)\left(z_{i}-\frac{1}{q} y_{j}\right)} \prod_{j=1}^{m} \prod_{i=1}^{k}\left(y_{j}-\sqrt{q t} x_{i}\right) \prod_{j=1}^{l} \prod_{i=1}^{n}\left(z_{j}-\sqrt{q t} w_{i}\right)
\end{aligned}
$$

The coupling between two DF integral (one with parameters $(q, t)$ and the other with $\left.\left(t^{-1}, q^{-1}\right)\right)$ in the last line in eq. (3.10) is due to the interaction (3.9).

From the field theory point of view the integral (3.10) describes two $3 d$ theories

1. $\mathcal{N}=2_{t}^{*} \mathrm{U}(m)$ gauge theory with $n$ fundamental multiplets in the $\Omega$-background with parameter $q$ and axial mass deformation $t$,

2. $\mathcal{N}=2_{q^{-1}}^{*} \mathrm{U}(l)$ gauge theory with $k$ fundamental multiplets in the $\Omega$-background with parameter $t^{-1}$ and axial mass deformation $q^{-1}$.

These two theories are coupled through a $1 d$ interaction term. This setup was described in $[57]$. 
The situation with more than two violet horizontal lines is similar: there are twice as many types of screening charges as there were in section 2.4. All these screening charges commute with the action of $q W_{N}$ algebra. The corresponding field theory is a pair of $3 d$ theories coupled thorough $1 d$ interaction.

\subsection{Gluing different Fock spaces. Fermionic screenings}

The final logical step in our formalism is to stack together horizontal lines of different colors. Let us start with two lines, e.g. violet and red. Between them we can stretch dashed lines of the color different from both horizontal lines. There is, therefore, only one choice, blue, which produces the screening current $S_{q}^{q, t^{-1} \mid q, t / q}(w)$

$$
\longleftarrow \begin{gathered}
\longleftarrow y \\
\vdots \\
\vdots
\end{gathered}=\int_{-\infty}^{\infty} d_{q} y \Phi_{\Phi_{q, t^{-1}}^{q}(y)}^{* q, t / q}(y)=\int_{-\infty}^{\infty} d_{q} y S_{q}^{q, t^{-1} \mid q, t / q}(y) .
$$

Evaluating the screening current explicitly we get

$$
\begin{aligned}
S_{q}^{q, t^{-1} \mid q, t / q}(y)= & e^{-\left(\epsilon_{1}+\epsilon_{2}\right) Q_{1}-\epsilon_{2} Q_{2}} y^{\frac{\epsilon_{1}+\epsilon_{2}-P_{1}+P_{2}}{\epsilon_{1}}} \times \\
& \times \exp \left[\sum_{n \geq 1} \frac{y^{n}}{n\left(1-q^{n}\right)}\left(t^{-\frac{n}{2}}\left(1-(t / q)^{n}\right) a_{-n}^{(1)}-\left(1-t^{-n}\right) a_{-n}^{(2)}\right)\right] \times \\
& \times \exp \left[\sum_{n \geq 1} \frac{y^{-n}}{n\left(1-q^{-n}\right)}\left(-t^{-\frac{n}{2}}\left(1-(q / t)^{n}\right) a_{n}^{(1)}+\left(1-t^{n}\right) a_{n}^{(2)}\right)\right]
\end{aligned}
$$

where the bosons satisfy

$$
\left[a_{n}^{(1)}, a_{m}^{(1)}\right]=n \frac{1-q^{|n|}}{1-\left(\frac{q}{t}\right)^{|n|}} \delta_{n+m, 0}, \quad\left[a_{n}^{(2)}, a_{m}^{(2)}\right]=n \frac{1-q^{|n|}}{1-t^{|n|}} \delta_{n+m, 0},
$$

An explicit calculation shows that the currents $S_{q}^{q, t^{-1} \mid q, t / q}(y)$ anticommute. Such fermionic screenings appear naturally in the context of $W$-algebras associated with superalgebras [52]. In particular, on two horizontal lines of different colors, DIM algebra is expected to act as a $W$-algebra associated to superalgebra $\mathfrak{g l}_{1 \mid 1}$ (somewhat similarly to the case of two Fock spaces of the same color where a product of $q$-Virasoro and Heisenberg algebras, associated to $\mathfrak{g l}_{2}$ acts). Having the fermionic screening we can build interesting DF-type integrals ${ }^{5}$ and the corresponding $3 d$ quiver gauge theories. Both of them are associated to Dynkin diagrams of superalgebras. Let us write down the simplest example, corresponding to the network

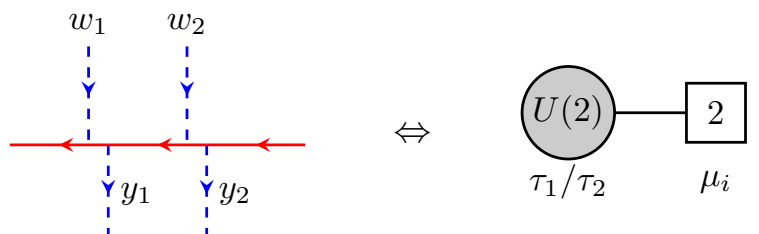

\footnotetext{
${ }^{5}$ Unfortunately the name superintegral is already taken.
} 
The vacuum matrix element of (3.14) is

$$
\oint_{\mathcal{C}_{1,1}}\left(y_{1} y_{2}\right)^{\log _{q} \frac{u_{2}}{u_{1}}+2 \beta-3} \frac{\Delta_{2}(\vec{y})}{\bar{\Delta}_{2,2}^{(q, q / t)}(\vec{y}, \vec{w})},
$$

where $\Delta_{2}(\vec{y})$ is the square of the ordinary (i.e. not $(q, t)$-deformed) Vandermonde determinant:

$$
\Delta_{m}(\vec{y})=\prod_{i \neq j}^{m}\left(1-\frac{y_{i}}{y_{j}}\right) .
$$

This gives us a hint at what a $3 d \mathcal{N}=2^{*}$ theory associated to superquiver looks like: the gauge node (the only one in the example, painted graw in (3.14)), corresponding to the fermionic root has trivial axial deformation parameter, as if $\mathcal{N}=4$ supersymmetry was unbroken. More generally, for several bosonic roots separated by a fermionic one, the axial mass parameter $m_{A}$ changes sign along the quiver: e.g. it is $t$ for the bosonic nodes to the left of the fermionic node, and $\frac{q}{t}$ for the bosonic nodes to the right of the fermionic one. On the fermionic node we effectively have the theory with $t=q$.

Notice that the setup with horizontal solid lines of different colors which we consider in this section is different from that with differently colored vertical dashed lines from section 3.1. While the former gives a peculiar gauge theory on a $3 d$ space $\mathbb{R}^{2} \times_{q} S^{1}$, the latter produces a pair of $3 d$ theories, one living on $\mathbb{R}^{2} \times{ }_{q} S^{1}$ and another on $\mathbb{R}^{2} \times{ }_{t^{-1}} S^{1}$, coupled through some $1 d$ degrees of freedom living on $S^{1}$. Our algebraic formalism incorporates both of these setups, so it is natural to ask if there might be a relation between the two theories, but we currently make no claims in this regard.

Finally, as an exercise we draw a colorful picture incorporating various screenings we have obtained:

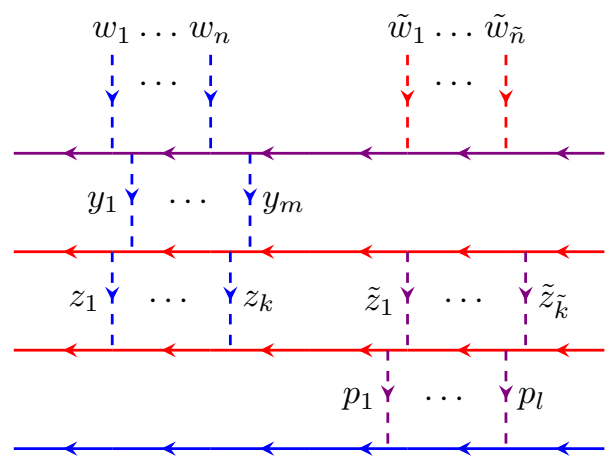

The resulting $q W$-algebra should be associated to a certain reduction of a sum of superalgebras $\mathfrak{g l}_{1 \mid 2} \oplus \mathfrak{g l}_{2 \mid 1}$. Indeed, if we for a moment exclude the blue horizontal line from the picture (3.17) then according to [52] we should get $q W\left[\mathfrak{g l}_{1 \mid 2}\right]$, while if we delete the violet horizontal line, we should get $q W\left[\mathfrak{g l}_{2 \mid 1}\right]$. Having all three types of horizontal lines should probably give a certain reduction of $q W\left[\mathfrak{g l}_{1 \mid 2}\right] \oplus q W\left[\mathfrak{g l}_{2 \mid 1}\right]$.

One can analyze what sort of gauge theory corresponds to the network (3.17) by cutting the picture into smaller pieces, which we have already discussed. Considering the pieces involving dashed lines of a given color separated horizontally from each other we observe that the corresponding gauge theory consists of three $3 d$ theories each living in its own $S^{1} \times \mathbb{R}^{2}$ space with $\Omega$-background parameters $q, t^{-1}$ and $\frac{t}{q}$ respectively: 
1. Supersymmetric quiver theory associated to the algebra $\mathfrak{g l}_{2 \mid 1}$ on $S^{1} \times_{q} \mathbb{R}^{2}$ with axial mass parameters $t$ and $\frac{q}{t}$.

2. Supersymmetric quiver theory associated to the algebra $\mathfrak{g l}_{1 \mid 1}$ on $S^{1} \times_{\frac{t}{q}} \mathbb{R}^{2}$ with axial mass parameters $q^{-1}$ and $t$.

3. Theory of free multiplets on $S^{1} \times_{t^{-1}} \mathbb{R}^{2}$ with axial mass deformation $q^{-1}$.

Each of the theories 1, 2 and 3 have already appeared in our discussions, and we have seen that they indeed correspond to pieces of the picture (3.17). Pairs of theories $(1,2)$ and $(2,3)$ are coupled by $1 d$ interaction terms, which can be deduced from the form of the partition function corresponding (which in turn can be computed by normal ordering the intertwining operators).

\section{Ruijsenaars Hamiltonians and their supersymmetric versions}

In this section we prove that the networks we have constructed are the eigenfunctions of (supersymmetric) Ruijsenaars Hamiltonians acting on the spectral parameters of the external dashed lines.

We first consider a simple example and then argue that the statement actually holds for a larger class of networks. Our example is the $T[\mathrm{SU}(2)]$ theory. The proof here is already well-known, but we rederive it using the intertwining property of Higgsed networks to show that it is automatic in our approach. Consider the action of DIM element $\Delta^{3}\left(x_{0}^{+}\right)$ on the corresponding network (2.23):

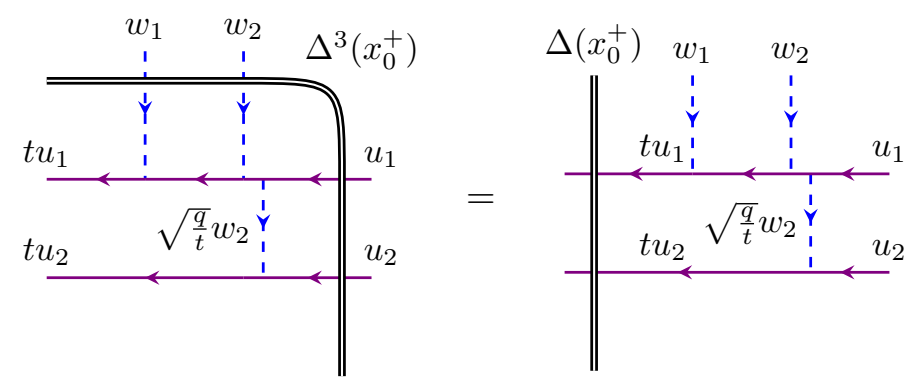

where the equality follows from the definition of the intertwining operator. Using the coproduct from section A.2 we find that on the tensor product of two vector representation and two Fock representations one has

$$
\begin{aligned}
& \left.\Delta^{3}\left(x_{0}^{+}\right)\right|_{\mathcal{V}_{w_{1}}^{q} \otimes \mathcal{V}_{w_{2}}^{q} \otimes \mathcal{F}_{u_{1}}^{q, t^{-1}} \otimes \mathcal{F}_{u_{1}}^{q, t^{-1}}}= \\
& =\left.\Delta\left(x_{0}^{+}\right)\right|_{\mathcal{V}_{w_{1}}^{q} \otimes \mathcal{V}_{w_{2}}^{q}}+\left.\left.\oint_{\mathcal{C}} \frac{d z}{z}\left(\psi^{-}(z) \otimes \psi^{-}(z)\right)\right|_{\mathcal{V}_{w_{1}}^{q} \otimes \mathcal{V}_{w_{2}}^{q}} \otimes \Delta\left(x^{+}(z)\right)\right|_{\mathcal{F}_{u_{1}}^{q, t^{-1}} \otimes \mathcal{F}_{u_{1}}^{q, t^{-1}}}
\end{aligned}
$$

where the integration contour $\mathcal{C}$ is a small contour around $z=0$. Eq. (4.2) gives the DIM action featuring in the 1.h.s. of eq. (4.1).

DIM action in the r.h.s. of eq. (4.1) is just $\left.\Delta\left(x^{+}(z)\right)\right|_{\mathcal{F}_{u_{1}}^{q, t^{-1}} \otimes \mathcal{F}_{u_{1}}^{q, t^{-1}}}$ (see (A.5)). Sandwiching both sides of eq. (4.1) between the vacuum states, we find that 


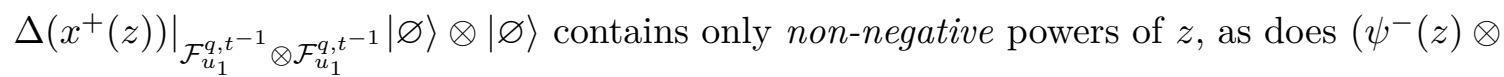
$\left.\psi^{-}(z)\right)\left.\right|_{\mathcal{V}_{w_{1}}^{q} \otimes \mathcal{V}_{w_{2}}^{q}}$ (see the representations in section A.3 and A.4). Thus, the integral in eq. (4.2) is equal to the zero mode of $\left.\Delta\left(x^{+}(z)\right)\right|_{\mathcal{F}_{u_{1}}^{q, t^{-1}} \otimes \mathcal{F}_{u_{1}}^{q, t^{-1}}}|\varnothing\rangle \otimes|\varnothing\rangle$, i.e. to $\frac{\left(u_{1}+u_{2}\right)}{\left(1-q^{-1}\right)(1-t)}|\varnothing\rangle \otimes|\varnothing\rangle$. We have

$$
\begin{aligned}
& \left.\Delta^{3}\left(x_{0}^{+}\right)\right|_{\mathcal{V}_{w_{1}}^{q} \otimes \mathcal{V}_{w_{2}}^{q} \otimes \mathcal{F}_{u_{1}}^{q, t^{-1}} \otimes \mathcal{F}_{u_{1}}^{q, t^{-1}}}\left|w_{1}\right\rangle \otimes\left|w_{2}\right\rangle \otimes|\varnothing\rangle \otimes|\varnothing\rangle= \\
& =-\frac{1}{1-q^{-1}}\left|q w_{1}\right\rangle \otimes\left|w_{2}\right\rangle \otimes|\varnothing\rangle \otimes|\varnothing\rangle+ \\
& \quad-\frac{1}{1-q^{-1}} \frac{\left(1-t \frac{w_{2}}{w_{1}}\right)\left(1-\frac{q}{t} \frac{w_{2}}{w_{1}}\right)}{\left(1-\frac{w_{2}}{w_{1}}\right)\left(1-q \frac{w_{2}}{w_{1}}\right)}\left|w_{1}\right\rangle \otimes\left|q w_{2}\right\rangle \otimes|\varnothing\rangle \otimes|\varnothing\rangle+ \\
& \quad+\frac{u_{1}+u_{2}}{\left(1-q^{-1}\right)(1-t)}\left|w_{1}\right\rangle \otimes\left|w_{2}\right\rangle \otimes|\varnothing\rangle \otimes|\varnothing\rangle
\end{aligned}
$$

In the r.h.s. of eq. (4.1) we find that $\langle\varnothing| \otimes\langle\varnothing| \Delta\left(x_{0}^{+}\right)$also reduces to zero modes, which in this case are $\langle\varnothing| \otimes\langle\varnothing| \frac{\left(t u_{1}+t u_{2}\right)}{\left(1-q^{-1}\right)(1-t)}$. Substituting both sides of eq. (4.1) and denoting the vacuum matrix element of the network by $\psi(\vec{w}, \vec{u})$ we can write

$$
\left(q^{w_{1} \partial_{w_{1}}}+\frac{\left(1-t \frac{w_{2}}{w_{1}}\right)\left(1-\frac{q}{t} \frac{w_{2}}{w_{1}}\right)}{\left(1-\frac{w_{2}}{w_{1}}\right)\left(1-q \frac{w_{2}}{w_{1}}\right)} q^{w_{2} \partial_{w_{2}}}\right) \psi(\vec{w}, \vec{u})=\left(u_{1}+u_{2}\right) \psi(\vec{w}, \vec{u})
$$

Factoring the flipping field contributions (see eq. (2.22)) out of $\psi(\vec{w}, \vec{u})$ we find that the function

$$
\tilde{\psi}(\vec{w}, \vec{u})=\frac{\left(t \frac{w_{1}}{w_{2}} ; q\right)_{\infty}}{\left(\frac{w_{1}}{w_{2}} ; q\right)_{\infty}} \psi(\vec{w}, \vec{u})
$$

is an eigenfunction of the Ruijsenaars Hamiltonian:

$$
H_{1} \tilde{\psi}(\vec{w}, \vec{u})=\left(\frac{1-t \frac{w_{1}}{w_{2}}}{1-\frac{w_{1}}{w_{2}}} q^{w_{1} \partial_{w_{1}}}+\frac{1-t \frac{w_{2}}{w_{1}}}{1-\frac{w_{2}}{w_{1}}} q^{w_{2} \partial_{w_{2}}}\right) \tilde{\psi}(\vec{w}, \vec{u})=\left(u_{1}+u_{2}\right) \tilde{\psi}(\vec{w}, \vec{u}) .
$$

The argument of intertwining is completely general, thus any Higgsed network of the form we have considered is an eigenfunction of the Ruijsenaars Hamiltonians with eigenvalues determined by the spectral parameters of the horizontal lines.

Let us finally mention the supersymmetric generalization of the Ruijsenaars system, which is obtained by considering the network (3.10). In this case there are two types of DIM action in the vertical representations, which give rise to two coupled Ruijsenaars Hamiltonians:

$$
\begin{aligned}
H_{1}^{\text {super }}= & \sum_{i=1}^{k} \prod_{a=1}^{n} \frac{x_{i}-q w_{a}}{x_{i}-w_{a}} \prod_{j \neq i} \frac{t x_{i}-x_{j}}{x_{i}-x_{j}} q^{x_{i} \partial_{x_{i}}}+ \\
& +\frac{1-q^{-1}}{1-t} \sum_{i=1}^{n} \prod_{a=1}^{k} \frac{w_{i}-t^{-1} x_{a}}{w_{i}-x_{a}} \prod_{j \neq i} \frac{q^{-1} w_{i}-w_{j}}{w_{i}-w_{j}} t^{-w_{i} \partial_{w_{i}}}
\end{aligned}
$$


The interaction terms in the Hamiltonian are due to the nontriviality of the DIM coproduct. Denoting the value of the vacuum matrix element of (3.10) (stripped off the flipping fields) by $\tilde{\psi}(\vec{w}, \vec{x}, \vec{u})$ we get

$$
H_{1}^{\text {super }} \tilde{\psi}(\vec{w}, \vec{x}, \vec{u})=\frac{1}{1-t}\left(\left(1-t^{k-l} q^{m-n}\right) u_{1}+\left(1-t^{l} q^{m}\right) u_{2}\right) \tilde{\psi}(\vec{w}, \vec{x}, \vec{u}) .
$$

Such system was considered in [62]. ${ }^{6}$ Our formalism provides explicit integral formulas for the solutions of the supersymmetric Ruijsenaars system and of the corresponding supersymmetric Macdonald polynomials (and, in the $t \rightarrow q$ limit, supersymmetric Schur functions).

\section{Conclusions and discussions}

We have introduced a version of the network formalism based on the DIM algebra intertwiners. It can be thought of as an analogue of the refined topological vertex formalism for the case incorporating not only a network of five-branes of Type IIB, but also D3 branes. On the field theory side it provides a way to understand partition functions (holomorphic blocks) of $3 d \mathcal{N}=2^{*}$ quiver gauge theories. In particular, it gives a constructive definition of the partition functions for the quiver theories with quivers having the form of Dynkin diagrams of superalgebras and gives a transparent proof of the fact that the partition functions are eigenfunctions of (supersymmetric) Ruijsenaars-Schneider Hamiltonians. On the algebraic side our construction naturally produces screenings for the $q W$-algebras, including those associated with superalgebras.

There are many directions along which one can extend the approach presented here. For example we have just started studying the $3 d$ theories corresponding to superquivers ${ }^{7}$ - there is a wealth of interesting examples which can be built rather straightforwardly with a simple set of building blocks, as e.g. the picture (3.17) demonstrates. Some of the networks we have introduced can be compactified, i.e. drawn on a cylinder or torus instead of a plane. This should give rise to explicit description of the eigenfunctions of quantum elliptic and double elliptic systems [79-84]. It would be interesting to study the monodromy problems for the networks we have introduce - they should be described by the elliptic stable envelopes theory [85-88].

\section{Acknowledgments}

The author would like to thank B. Feigin for a discussion. The author is supported by the RSF grant 18-71-10073.

\footnotetext{
${ }^{6}$ Their notation differs from the notation here: $(q, t)_{[62]}=\left(q, t^{-1}\right)_{\text {here }}$.

${ }^{7}$ We should emphasize that there are two a priori unrelated ways of incorporating supergroups into the gauge theories. The first way is to consider supergroups as gauge groups [74-78]. The second is to consider quiver gauge theories with quivers having special "fermionic" vertices, corresponding to Dynkin diagrams of superaglebras $[58,59]$. It is the latter approach which is incorporated into our formalism (see section 3.2).
} 


\section{A DIM algebra and its representations}

For the sake of completeness in this appendix we list the relevant formulas from the theory of DIM algebras, mostly taken from [6, 60, 61].

\section{A.1 The algebra}

DIM algebra $U_{q, t}\left(\widehat{\widehat{\mathfrak{g l}}}_{1}\right)$ is generated by the currents $x^{ \pm}(z)=\sum_{n \in \mathbb{Z}} x_{n}^{ \pm} z^{-n}, \psi^{ \pm}(z)=$ $\sum_{n \gtreqless 0} \psi_{n}^{ \pm} z^{-n}$ and a central element $\gamma$ subject to the relations ${ }^{8}$

$$
\begin{aligned}
& {\left[\psi^{ \pm}(z), \psi^{ \pm}(w)\right]=0} \\
& \psi^{+}(z) \psi^{-}(w)=\frac{g\left(\gamma^{\frac{w}{z}}\right)}{g\left(\gamma^{-1} \frac{w}{z}\right)} \psi^{-}(w) \psi^{+}(z) \\
& \psi^{+}(z) x^{ \pm}(w)=g\left(\gamma^{\mp \frac{1}{2}} \frac{w}{z}\right)^{\mp 1} x^{ \pm}(w) \psi^{+}(z), \quad \psi^{-}(z) x^{ \pm}(w)=g\left(\gamma^{\mp \frac{1}{2}} \frac{w}{z}\right)^{ \pm 1} x^{ \pm}(w) \psi^{-}(z) \\
& {\left[x^{+}(z), x^{-}(w)\right]=\frac{1}{G^{-}(1)}\left(\delta\left(\gamma^{-1} \frac{z}{w}\right) \psi^{+}\left(\gamma^{\frac{1}{2}} w\right)-\delta\left(\gamma \frac{z}{w}\right) \psi^{-}\left(\gamma^{-\frac{1}{2}} w\right)\right),} \\
& G^{\mp}\left(\frac{z}{w}\right) x^{ \pm}(z) x^{ \pm}(w)=G^{ \pm}\left(\frac{z}{w}\right) x^{ \pm}(w) x^{ \pm}(z),
\end{aligned}
$$

where $\delta(x)=\sum_{n \in \mathbb{Z}} x^{n}$ and the "structure functions"9 of the algebra are given by $G^{ \pm}(x)=$ $\left(1-q^{ \pm 1} x\right)\left(1-t^{\mp 1} x\right)\left(1-t^{ \pm 1} q^{\mp 1} x\right)$ and $g(x)=\frac{G^{+}(x)}{G^{-}(x)}$. Notice that $g\left(\frac{1}{x}\right)=\frac{1}{g(x)}$ and in particular $G^{+}(1)=G^{-}(1)$. The ratio of the zero modes $\frac{\psi_{0}^{-}}{\psi_{0}^{+}}=\gamma_{\perp}^{2}$ also turns out to be central. ${ }^{10}$ There are also Serre relations for triple commutators of $x^{+}(z)$ and $x^{-}(z)$, which we will not write down here.

Notice that the relations of the algebra are manifestly symmetric under the action of $\mathfrak{S}_{3}$ group permuting the triplet of deformation parameters $\left(q, t^{-1}, t / q\right)$. Only part of this symmetry will be retained by the representations which we are going to consider. By this we mean that some permutations will not affect a representation, while others will turn a representation into an isomorphic one.

DIM algebra respects two gradings, $d$ and $d^{\perp} . d$ counts the number of the Laurent mode of a current, so that $d\left(x_{n}^{ \pm}\right)=d\left(\psi_{n}^{ \pm}\right)=n$, while $d^{\perp}$ is a "perpendicular" grading defined as $d^{\perp}\left(x_{n}^{ \pm}\right)= \pm 1, d^{\perp}\left(\psi_{n}^{ \pm}\right)=0$.

There is an extra symmetry of the DIM algebra, which is not manifest in the definition (A.1)-(A.4) - the $\mathrm{SL}(2, \mathbb{Z})$ automorphism group (the most tricky part of it is the action of the $S$-element, known as the Miki automorphism). We will not define the action of this symmetry on the currents explicitly. It will be enough for us to visualize it as an action of $\mathrm{SL}(2, \mathbb{Z})$ on the double grading lattice $\left(d, d^{\perp}\right) \in \mathbb{Z}^{2}$ and on the doublet of central charges $\left(\gamma^{2}, \gamma_{\perp}^{2}\right)$, so that e.g. $x_{0}^{+}$is turned into $\psi_{-1}^{-}$by the $S$ element.

\footnotetext{
${ }^{8}$ This definition differs from the definition of [6] by the rescaling of the generators $x_{\text {our }}^{ \pm}(z)=(1-$ $\left.q^{\mp 1}\right)^{-1}\left(1-t^{ \pm 1}\right)^{-1} x_{\mathrm{AFS}}^{ \pm}(z)$, while keeping $\psi_{\mathrm{our}}^{ \pm}(z)=\psi_{\mathrm{AFS}}^{ \pm}(z)$.

${ }^{9}$ Curiously, they also appear as factorized scattering matrices of some $2 d$ integrable models. It is tempting to try to understand DIM algebra as a version of the Zamolodchikov-Fateev algebra of such models.

${ }^{10}$ The product of the zero modes $\psi_{0}^{-} \psi_{0}^{+}$is central too, but can be eliminated by an overall rescaling of all the currents.
} 


\section{A.2 The coproduct}

DIM algebra can be endowed with a coproduct $\Delta$, which acts on the generators as follows:

$$
\begin{aligned}
& \Delta\left(x^{+}(z)\right)=x^{+}(z) \otimes 1+\psi^{-}\left(\gamma_{(1)}^{\frac{1}{2}} z\right) \otimes x^{+}\left(\gamma_{(1)} z\right) \\
& \Delta\left(x^{-}(z)\right)=x^{-}\left(\gamma_{(2)} z\right) \otimes \psi^{+}\left(\gamma_{(2)}^{\frac{1}{2}} z\right)+1 \otimes x^{-}(z), \\
& \Delta\left(\psi^{ \pm}(z)\right)=\psi^{ \pm}\left(\gamma_{(2)}^{ \pm \frac{1}{2}} z\right) \otimes \psi^{ \pm}\left(\gamma_{(1)}^{\mp \frac{1}{2}} z\right) .
\end{aligned}
$$

where $\gamma_{(1)}\left(\right.$ resp. $\left.\gamma_{(2)}\right)$ denotes the central charge of the first (resp. second) representation in the tensor product. Since the currents are formal infinite Laurent series, the products in the r.h.s. may require regularization for some representations. We will not encounter this problem for the representations we will consider.

The coproduct $\Delta$ respects the $\mathfrak{S}_{3}$ permutation symmetry of the DIM algebra but is not invariant under the action of $\mathrm{SL}(2, \mathbb{Z})$. In fact there is an infinite number of coproducts, parametrized by irrational slopes on the $2 d$ plane. All these coproducts are related to each other by nontrivial Drinfeld twists (see [15, 16], and for a more geometric view also [17]).

\section{A.3 Horizontal Fock representation}

There is a representation of DIM algebra on the Fock space $\mathcal{F}_{u}^{(1,0), q, t^{-1}}$, generated by the action of creation operators $a_{-n}, n \in \mathbb{Z}_{>0}$ on the vacuum vector $|\varnothing, u\rangle$. The states of $\mathcal{F}_{u}^{q, t^{-1}}$ are therefore labelled by Young diagrams. The combination of indices $(1,0)$ and $q, t^{-1}$ of the representation is used to denote the "direction" (horizontal) and "length" of its central charge vector $\left(\gamma^{2}, \gamma_{\perp}^{2}\right)=\left((t / q)^{1},(t / q)^{0}\right)=(t / q, 1)$ respectively. We will usually omit the index $(1,0)$, when it is clear what is the direction of the central charge vector. Creation and annihilation operators satisfy the commutation relations

$$
\left[a_{n}, a_{m}\right]=n \frac{1-q^{|n|}}{1-t^{|n|}} \delta_{n+m, 0}
$$

while the zero modes $P$ and $Q$ commute with $a_{n}$ and satisfy the standard Heisenberg commutation relations

$$
[P, Q]=1
$$

The zero modes act on the vacuum vector as follows:

$$
P|\varnothing, u\rangle=\ln u|\varnothing, u\rangle, \quad e^{\alpha Q}|\varnothing, u\rangle=\left|\varnothing, e^{\alpha} u\right\rangle .
$$


The action of the DIM generators is given by the following vertex operators:

$$
\begin{aligned}
x^{+}(z)= & \left(1-q^{-1}\right)^{-1}(1-t)^{-1} e^{P} \exp \left[\sum_{n \geq 1} \frac{z^{n}}{n}\left(1-t^{-n}\right) a_{-n}\right] \exp \left[-\sum_{n \geq 1} \frac{z^{-n}}{n}\left(1-t^{n}\right) a_{n}\right], \\
x^{-}(z)= & (1-q)^{-1}\left(1-t^{-1}\right)^{-1} e^{-P} \exp \left[-\sum_{n \geq 1} \frac{z^{n}}{n}\left(1-t^{-n}\right)\left(\frac{t}{q}\right)^{\frac{n}{2}} a_{-n}\right] \times \\
& \times \exp \left[-\sum_{n \geq 1} \frac{z^{-n}}{n}\left(1-t^{n}\right)\left(\frac{t}{q}\right)^{\frac{n}{2}} a_{n}\right], \\
\psi^{+}(z)= & \exp \left[-\sum_{n \geq 1} \frac{z^{-n}}{n}\left(1-t^{n}\right)\left(1-\left(\frac{t}{q}\right)^{n}\right)\left(\frac{q}{t}\right)^{\frac{n}{4}} a_{n}\right], \\
\psi^{-}(z)= & \exp \left[\sum_{n \geq 1} \frac{z^{n}}{n}\left(1-t^{-n}\right)\left(1-\left(\frac{t}{q}\right)^{n}\right)\left(\frac{q}{t}\right)^{\frac{n}{4}} a_{-n}\right] .
\end{aligned}
$$

The representation $\mathcal{F}_{u}^{q, t^{-1}}$ is invariant under the exchange of $q$ and $t^{-1}$ deformation parameters. To see this we notice that the exchange $q \leftrightarrow t^{-1}$ in the vertex operators (A.11)-(A.13) is equivalent to the rescaling of the creation and annihilation operators:

$$
a_{n}^{\left(q, t^{-1}\right)}=\frac{1-q^{-n}}{1-t^{n}} a_{n}^{\left(t^{-1}, q\right)} .
$$

Notice in particular, that $a_{n}^{\left(t^{-1}, q\right)}$ satisfy

$$
\left[a_{n}^{\left(t^{-1}, q\right)}, a_{m}^{\left(t^{-1}, q\right)}\right]=n \frac{1-t^{-|n|}}{1-q^{-|n|}} \delta_{m+n, 0}
$$

as they should. This symmetry might be familiar from the theory of Macdonald polynomials $M_{Y}^{\left(q, t^{-1}\right)}\left(a_{-n}^{\left(q, t^{-1}\right)}\right)$, in which it corresponds to the transposition of the Young diagram $Y$. As we have mentioned above, this $\mathbb{Z}_{2}$ symmetry of the Fock representation is part of the larger $\mathfrak{S}_{3}$ permutation symmetry of the DIM algebra. The remaining elements of $\mathfrak{S}_{3}$ transform $\mathcal{F}_{u}^{q, t^{-1}}$ into two more horizontal Fock representations $\mathcal{F}_{u}^{q, t / q}$ and $\mathcal{F}_{u}^{t^{-1}, t / q}$, obtained from (A.11)-(A.13) by permuting the parameters of the algebra. Their central charge vectors $\left(\gamma^{2}, \gamma_{\perp}^{2}\right)$ are $\left(t^{-1}, 1\right)$ and $(q, 1)$ respectively.

Fock representation with different slope, e.g. a vertical one $\mathcal{F}_{u}^{(0,1), q, t^{-1}}$ can be obtained by the action of the elements of $\operatorname{SL}(2, \mathbb{Z})$ automorphism group on $\mathcal{F}_{u}^{(1,0), q, t^{-1}}$. We will not need these representations for the construction presented in the main text, so we omit their explicit definition. For more information on the action of $\mathrm{SL}(2, \mathbb{Z})$ and its implications see $[8,27-35]$.

\section{A.4 Vertical vector representation}

The vertical vector representation $\mathcal{V}_{w}^{q}$ has trivial central charges $\left(\gamma^{2}, \gamma_{\perp}^{2}\right)=(1,1)$. It can be understood as a kind of evaluation representation for the currents $x^{ \pm}(z), \psi^{ \pm}(z)$, similar 
to evaluation representations of (quantum) affine algebra. There are two equivalent ways to view $\mathcal{V}_{w}^{q}$ : either as an infinite dimensional representation parametrized by $w$ with basis $|n, w\rangle, n \in \mathbb{Z}$, or as a representation on the space of functions of $w$ with state $|n, w\rangle$ corresponding to function $\left|q^{n} w\right\rangle$. In the first case the action of the DIM currents is

$$
\begin{aligned}
x^{+}(z)|w, n\rangle & =-\frac{1}{1-q^{-1}} \delta\left(\frac{q^{n} w}{z}\right)|w, n+1\rangle, \\
x^{-}(z)|w, n\rangle & =\frac{1}{1-q} \delta\left(\frac{q^{n-1} w}{z}\right)|w, n-1\rangle, \\
\psi^{+}(z)|w, n\rangle & =\frac{\left(1-\frac{t}{q} \frac{q^{n} w}{z}\right)\left(1-\frac{1}{t} \frac{q^{n} w}{z}\right)}{\left(1-\frac{q^{n} w}{z}\right)\left(1-\frac{1}{q} \frac{q^{n} w}{z}\right)}|w, n\rangle, \\
\psi^{-}(z)|w, n\rangle & =\frac{\left(1-\frac{q}{t} \frac{z}{q^{n} w}\right)\left(1-t \frac{z}{q^{n} w}\right)}{\left(1-\frac{z}{q^{n} w}\right)\left(1-q \frac{z}{q^{n} w}\right)}|w, n\rangle .
\end{aligned}
$$

In the second view the currents act on functions $|w\rangle$ :

$$
\begin{aligned}
x^{+}(z)|w\rangle & =-\frac{1}{1-q^{-1}} \delta\left(\frac{w}{z}\right)|q w\rangle, \\
x^{-}(z)|w\rangle & =-\frac{1}{1-q} \delta\left(\frac{w}{q z}\right)\left|\frac{w}{q}\right\rangle, \\
\psi^{+}(z)|w\rangle & =\frac{\left(1-\frac{t}{q} \frac{w}{z}\right)\left(1-\frac{1}{t} \frac{w}{z}\right)}{\left(1-\frac{w}{z}\right)\left(1-\frac{1}{q} \frac{w}{z}\right)}|w\rangle, \\
\psi^{-}(z)|w\rangle & =\frac{\left(1-\frac{q}{t} \frac{z}{w}\right)\left(1-t \frac{z}{w}\right)}{\left(1-\frac{z}{w}\right)\left(1-q \frac{z}{w}\right)}|w\rangle .
\end{aligned}
$$

We will use both views interchangeably at our convenience.

The vector representation $V_{w}^{q}$ is manifestly symmetric with respect to the exchange of $t$ and $\frac{q}{t}$. The action of $\mathfrak{S}_{3}$ permutation symmetry of DIM algebra produces two more vector representations $\mathcal{V}_{w}^{t^{-1}}$ and $\mathcal{V}_{w}^{t / q}$ defined in an obvious way.

\section{A.5 Visualizing representations}

Let us also mention that both Fock and vector representations can be thought of as certain reductions of a more general MacMahon representation of central charge $(1, K)[63-67,72,73]$ with general $K \in \mathbb{C}$ and states labelled by plane partitions, i.e. $3 d$ Young diagrams (here we do not pay attention to the direction of the central charge vector, focusing only on its "magnitude"). As a mnemonic aid, one can view the $3 d$ partitions constituting the MacMahon representation as living in a $\mathbb{Z}_{>0}^{3}$ space with three coordinate axes associated with three parameters $\left(q, t^{-1}, t / q\right)$ of the DIM algebra. A reduction of the representation corresponds to restriction to a subset of plane partitions of specific form:

1. Fock representation $\mathcal{F}_{u}^{q, t^{-1}}$ contains plane partitions of unit thickness lying along the $\left(q, t^{-1}\right)$ plane inside $\mathbb{Z}_{>0}^{3}$, i.e. those that reduce to Young diagrams. It is evident 
geometrically, that $\mathcal{F}_{u}^{q, t^{-1}}$ is invariant under the exchange of $q$ and $t^{-1}$ axes and that this symmetry corresponds to the transposition of a Young diagram $Y$ labelling a state of the representation. There are three coordinate planes, and therefore three Fock representations.

2. Vector representation $\mathcal{V}_{w}^{q}$ can be visualized as single column diagrams towering in the direction associated to $q$. One needs to stretch one's imagination a little bit in this case, since columns of negative height are also allowed. Naturally, the representation is invariant with respect to the exchange of coordinate axes $t \leftrightarrow \frac{q}{t}$, which lie perpendicular to $q$. There are three species of vector representations, corresponding to three different orientations of the columns.

Open Access. This article is distributed under the terms of the Creative Commons Attribution License (CC-BY 4.0), which permits any use, distribution and reproduction in any medium, provided the original author(s) and source are credited.

\section{References}

[1] J.-t. Ding and K. Iohara, Generalization and deformation of Drinfeld quantum affine algebras, Lett. Math. Phys. 41 (1997) 181 [INSPIRE].

[2] K. Miki, $A(q, \gamma)$ analog of the $W_{1+\infty}$ algebra, J. Math. Phys. 48 (2007) 123520.

[3] L.F. Alday, D. Gaiotto and Y. Tachikawa, Liouville Correlation Functions from Four-dimensional Gauge Theories, Lett. Math. Phys. 91 (2010) 167 [arXiv:0906.3219] [INSPIRE].

[4] N. Wyllard, $A(N-1)$ conformal Toda field theory correlation functions from conformal $N=2$ $\mathrm{SU}(N)$ quiver gauge theories, JHEP 11 (2009) 002 [arXiv: 0907.2189] [INSPIRE].

[5] A. Mironov and A. Morozov, On AGT relation in the case of U(3), Nucl. Phys. B $\mathbf{8 2 5}$ (2010) 1 [arXiv:0908. 2569] [INSPIRE].

[6] H. Awata, B. Feigin and J. Shiraishi, Quantum Algebraic Approach to Refined Topological Vertex, JHEP 03 (2012) 041 [arXiv: 1112.6074] [INSPIRE].

[7] H. Awata, B. Feigin, A. Hoshino, M. Kanai, J. Shiraishi and S. Yanagida, Notes on Ding-Iohara algebra and AGT conjecture, arXiv:1106.4088 [INSPIRE].

[8] Y. Zenkevich, Generalized Macdonald polynomials, spectral duality for conformal blocks and AGT correspondence in five dimensions, JHEP 05 (2015) 131 [arXiv:1412.8592] [INSPIRE].

[9] L. Bao, E. Pomoni, M. Taki and F. Yagi, M5-Branes, Toric Diagrams and Gauge Theory Duality, JHEP 04 (2012) 105 [arXiv:1112.5228] [INSPIRE].

[10] A. Mironov, A. Morozov, Y. Zenkevich and A. Zotov, Spectral Duality in Integrable Systems from AGT Conjecture, JETP Lett. 97 (2013) 45 [arXiv:1204.0913] [INSPIRE].

[11] A. Mironov, A. Morozov, B. Runov, Y. Zenkevich and A. Zotov, Spectral Duality Between Heisenberg Chain and Gaudin Model, Lett. Math. Phys. 103 (2013) 299 [arXiv:1206.6349] [INSPIRE].

[12] A. Mironov, A. Morozov, B. Runov, Y. Zenkevich and A. Zotov, Spectral dualities in XXZ spin chains and five dimensional gauge theories, JHEP 12 (2013) 034 [arXiv:1307.1502] [INSPIRE]. 
[13] A. Mironov, A. Morozov and Y. Zenkevich, Spectral duality in elliptic systems, six-dimensional gauge theories and topological strings, JHEP 05 (2016) 121 [arXiv: 1603.00304] [INSPIRE].

[14] A. Iqbal, C. Kozcaz and C. Vafa, The Refined topological vertex, JHEP 10 (2009) 069 [hep-th/0701156] [INSPIRE].

[15] H. Awata et al., Toric Calabi-Yau threefolds as quantum integrable systems. $\mathcal{R}$-matrix and $\mathcal{R} \mathcal{T} \mathcal{T}$ relations, JHEP 10 (2016) 047 [arXiv: 1608. 05351] [INSPIRE].

[16] H. Awata et al., Anomaly in RTT relation for DIM algebra and network matrix models, Nucl. Phys. B 918 (2017) 358 [arXiv:1611.07304] [InSPIRE].

[17] D. Maulik and A. Okounkov, Quantum Groups and Quantum Cohomology, arXiv: 1211.1287 [INSPIRE].

[18] H. Awata et al., Generalized Knizhnik-Zamolodchikov equation for Ding-Iohara-Miki algebra, Phys. Rev. D 96 (2017) 026021 [arXiv:1703.06084] [INSPIRE].

[19] H. Awata, H. Kanno, A. Mironov, A. Morozov, K. Suetake and Y. Zenkevich, $(q, t)-K Z$ equations for quantum toroidal algebra and Nekrasov partition functions on ALE spaces, JHEP 03 (2018) 192 [arXiv:1712.08016] [INSPIRE].

[20] N. Nekrasov, BPS/CFT correspondence: non-perturbative Dyson-Schwinger equations and qq-characters, JHEP 03 (2016) 181 [arXiv: 1512.05388] [INSPIRE].

[21] N. Nekrasov, BPS/CFT correspondence II: instantons at crossroads, moduli and compactness theorem, Adv. Theor. Math. Phys. 21 (2017) 503 [arXiv:1608.07272] [InSPIRE].

[22] N. Nekrasov, BPS/CFT Correspondence III: Gauge Origami partition function and qq-characters, Commun. Math. Phys. 358 (2018) 863 [arXiv:1701.00189] [InSPIRE].

[23] N. Nekrasov, BPS/CFT correspondence IV: sigma models and defects in gauge theory, Lett. Math. Phys. 109 (2019) 579 [arXiv:1711.11011] [INSPIRE].

[24] T. Kimura and V. Pestun, Quiver W-algebras, Lett. Math. Phys. 108 (2018) 1351 [arXiv: 1512.08533] [INSPIRE].

[25] T. Kimura and V. Pestun, Quiver elliptic W-algebras, Lett. Math. Phys. 108 (2018) 1383 [arXiv: 1608.04651] [INSPIRE].

[26] T. Kimura and V. Pestun, Fractional quiver W-algebras, Lett. Math. Phys. 108 (2018) 2425 [arXiv: 1705. 04410] [INSPIRE].

[27] A. Mironov, A. Morozov and Y. Zenkevich, Ding-Iohara-Miki symmetry of network matrix models, Phys. Lett. B 762 (2016) 196 [arXiv:1603.05467] [INSPIRE].

[28] H. Awata et al., Explicit examples of DIM constraints for network matrix models, JHEP 07 (2016) 103 [arXiv: 1604.08366] [INSPIRE].

[29] J.-E. Bourgine, M. Fukuda, Y. Matsuo, H. Zhang and R.-D. Zhu, Coherent states in quantum $\mathcal{W}_{1+\infty}$ algebra and qq-character for 5d Super Yang-Mills, PTEP 2016 (2016) 123B05 [arXiv: 1606.08020] [INSPIRE].

[30] J.-E. Bourgine, M. Fukuda, K. Harada, Y. Matsuo and R.-D. Zhu, (p, q)-webs of DIM representations, $5 d \mathcal{N}=1$ instanton partition functions and qq-characters, JHEP 11 (2017) 034 [arXiv: 1703.10759] [INSPIRE].

[31] J.-E. Bourgine, Webs of Quantum Algebra Representations in $5 d \mathcal{N}=1$ Super Yang-Mills, Springer Proc. Math. Stat. 263 (2017) 209 [INSPIRE]. 
[32] J.-E. Bourgine, M. Fukuda, Y. Matsuo and R.-D. Zhu, Reflection states in Ding-Iohara-Miki algebra and brane-web for D-type quiver, JHEP 12 (2017) 015 [arXiv:1709.01954] [INSPIRE].

[33] J.E. Bourgine and K. Zhang, A note on the algebraic engineering of $4 D \mathcal{N}=2$ super Yang-Mills theories, Phys. Lett. B 789 (2019) 610 [arXiv:1809.08861] [InSPIRE].

[34] J.-E. Bourgine, Fiber-base duality from the algebraic perspective, JHEP 03 (2019) 003 [arXiv: 1810.00301] [INSPIRE].

[35] A. Morozov and Y. Zenkevich, Decomposing Nekrasov Decomposition, JHEP 02 (2016) 098 [arXiv: 1510.01896] [INSPIRE].

[36] D. Gaiotto and M. Rapčák, Vertex Algebras at the Corner, JHEP 01 (2019) 160 [arXiv: 1703.00982] [INSPIRE].

[37] T. Procházka and M. Rapčák, Webs of W-algebras, JHEP 11 (2018) 109 [arXiv: 1711.06888] [INSPIRE].

[38] T. Procházka and M. Rapčák, $\mathcal{W}$-algebra modules, free fields, and Gukov-Witten defects, JHEP 05 (2019) 159 [arXiv:1808.08837] [INSPIRE].

[39] M. Rapcak, Y. Soibelman, Y. Yang and G. Zhao, Cohomological Hall algebras, vertex algebras and instantons, Commun. Math. Phys. 376 (2019) 1803 [arXiv:1810.10402] [INSPIRE].

[40] S. Pasquetti, Factorisation of $N=2$ Theories on the Squashed 3-Sphere, JHEP 04 (2012) 120 [arXiv: 1111.6905] [INSPIRE].

[41] C. Beem, T. Dimofte and S. Pasquetti, Holomorphic Blocks in Three Dimensions, JHEP 12 (2014) 177 [arXiv:1211.1986] [INSPIRE].

[42] A. Nedelin, S. Pasquetti and Y. Zenkevich, $T[\mathrm{SU}(N)]$ duality webs: mirror symmetry, spectral duality and gauge/CFT correspondences, JHEP 02 (2019) 176 [arXiv:1712.08140] [INSPIRE].

[43] F. Aprile, S. Pasquetti and Y. Zenkevich, Flipping the head of $T[\mathrm{SU}(N)]$ : mirror symmetry, spectral duality and monopoles, JHEP 04 (2019) 138 [arXiv:1812.08142] [INSPIRE].

[44] N. Dorey, T.J. Hollowood and D. Tong, The BPS spectra of gauge theories in two-dimensions and four-dimensions, JHEP 05 (1999) 006 [hep-th/9902134] [INSPIRE].

[45] A. Hanany and D. Tong, Vortices, instantons and branes, JHEP 07 (2003) 037 [hep-th/0306150] [INSPIRE].

[46] D. Tong, Monopoles in the Higgs phase, Phys. Rev. D 69 (2004) 065003 [hep-th/0307302] [INSPIRE].

[47] M. Shifman and A. Yung, NonAbelian string junctions as confined monopoles, Phys. Rev. D 70 (2004) 045004 [hep-th/0403149] [INSPIRE].

[48] A. Hanany and D. Tong, Vortex strings and four-dimensional gauge dynamics, JHEP 04 (2004) 066 [hep-th/0403158] [inSPIRE].

[49] N. Dorey, S. Lee and T.J. Hollowood, Quantization of Integrable Systems and a $2 d / 4 d$ Duality, JHEP 10 (2011) 077 [arXiv:1103.5726] [INSPIRE].

[50] H.-Y. Chen, N. Dorey, T.J. Hollowood and S. Lee, A New $2 d / 4 d$ Duality via Integrability, JHEP 09 (2011) 040 [arXiv: 1104.3021] [inSPIRE]. 
[51] A. Litvinov and L. Spodyneiko, On $W$ algebras commuting with a set of screenings, JHEP 11 (2016) 138 [arXiv: 1609.06271] [INSPIRE].

[52] M. Bershtein, B. Feigin and G. Merzon, Plane partitions with a "pit": generating functions and representation theory, Sel. Math. New Ser. 24 (2018) 21 [arXiv:1512.08779].

[53] D. Gaiotto and P. Koroteev, On Three Dimensional Quiver Gauge Theories and Integrability, JHEP 05 (2013) 126 [arXiv:1304.0779] [INSPIRE].

[54] M. Bullimore, H.-C. Kim and P. Koroteev, Defects and Quantum Seiberg-Witten Geometry, JHEP 05 (2015) 095 [arXiv: 1412.6081] [INSPIRE].

[55] P. Koroteev, P.P. Pushkar, A. Smirnov and A.M. Zeitlin, Quantum k-theory of Quiver Varieties and Many-Body Systems, arXiv:1705.10419 [INSPIRE].

[56] P. Koroteev and A.M. Zeitlin, qKZ/tRS Duality via Quantum K-Theoretic Counts, Math. Res. Lett. 28 (2021) 435 [arXiv: 1802.04463] [INSPIRE].

[57] F. Nieri, Y. Pan and M. Zabzine, 3d Expansions of $5 d$ Instanton Partition Functions, JHEP 04 (2018) 092 [arXiv: 1711.06150] [INSPIRE].

[58] N. Nekrasov, Superspin chains and supersymmetric gauge theories, JHEP 03 (2019) 102 [arXiv: 1811.04278] [INSPIRE].

[59] D. Orlando and S. Reffert, Relating Gauge Theories via Gauge/ Bethe Correspondence, JHEP 10 (2010) 071 [arXiv:1005.4445] [INSPIRE].

[60] B. Feigin, K. Hashizume, A. Hoshino, J. Shiraishi and S. Yanagida, A commutative algebra on degenerate $C P^{1}$ and Macdonald polynomials, J. Math. Phys. 50 (2009) 095215 [arXiv: 0904.2291].

[61] B. Feigin, A. Hoshino, J. Shibahara, J. Shiraishi and S. Yanagida, Kernel function and quantum algebras, arXiv:1002.2485.

[62] A.N. Sergeev and A.P. Veselov, Deformed Macdonald-Ruijsenaars operators and super Macdonald polynomials, arXiv:0707.3129.

[63] B. Feigin, E. Feigin, M. Jimbo, T. Miwa and E. Mukhin, Quantum continuous $\mathfrak{g l}_{\infty}$ : Semiinfinite construction of representations, Kyoto J. Math. 51 (2011) 337 [arXiv: 1002.3100].

[64] B. Feigin, E. Feigin, M. Jimbo, T. Miwa and E. Mukhin, Quantum continuous $\mathfrak{g l}_{\infty}$ : Tensor products of Fock modules and $W_{n}$ characters, arXiv:1002.3113 [INSPIRE].

[65] B. Feigin, M. Jimbo, T. Miwa and E. Mukhin, Quantum toroidal $\mathfrak{g l}_{1}$-algebra: Plane partitions, Kyoto J. Math. 52 (2012) 621 [arXiv:1110.5310].

[66] B. Feigin, M. Jimbo, T. Miwa and E. Mukhin, Representations of quantum toroidal $g l_{n}$, arXiv: 1204.5378

[67] B. Feigin, M. Jimbo, T. Miwa and E. Mukhin, Branching rules for quantum toroidal gl ${ }_{n}$, Adv. Math. 300 (2016) 229 [arXiv:1309.2147] [INSPIRE].

[68] B. Feigin, M. Jimbo, T. Miwa and E. Mukhin, Quantum toroidal $\mathfrak{g l}_{1}$ and Bethe ansatz, J. Phys. A 48 (2015) 244001 [arXiv: 1502.07194] [InSPIRE].

[69] B. Feigin, M. Jimbo, T. Miwa and E. Mukhin, Finite Type Modules and Bethe Ansatz for Quantum Toroidal $\mathfrak{g l}_{1}$, Commun. Math. Phys. 356 (2017) 285 [arXiv: 1603.02765] [INSPIRE]. 
[70] B. Feigin, M. Jimbo and E. Mukhin, Integrals of motion from quantum toroidal algebras, J. Phys. A 50 (2017) 464001 [arXiv:1705.07984] [INSPIRE].

[71] B. Feigin, M. Jimbo and E. Mukhin, The $\left(\mathfrak{g l}_{m}, \mathfrak{g l}_{n}\right)$ Duality in the Quantum Toroidal Setting, Commun. Math. Phys. 367 (2019) 455 [arXiv:1801.08433] [InSPIRE].

[72] Y. Zenkevich, 3d field theory, plane partitions and triple Macdonald polynomials, JHEP 06 (2019) 012 [arXiv:1712.10300] [INSPIRE].

[73] H. Awata, H. Kanno, A. Mironov, A. Morozov, K. Suetake and Y. Zenkevich, The MacMahon R-matrix, JHEP 04 (2019) 097 [arXiv:1810.07676] [INSPIRE].

[74] C. Vafa, Brane/anti-brane systems and $\mathrm{U}(N \mid M)$ supergroup, hep-th/0101218 [INSPIRE].

[75] R. Dijkgraaf, B. Heidenreich, P. Jefferson and C. Vafa, Negative Branes, Supergroups and the Signature of Spacetime, JHEP 02 (2018) 050 [arXiv: 1603.05665] [INSPIRE].

[76] T. Kimura and F. Nieri, Intersecting Defects and Supergroup Gauge Theory, arXiv:2105.02776 [INSPIRE].

[77] T. Kimura and V. Pestun, Super instanton counting and localization, arXiv:1905.01513 [INSPIRE].

[78] H.-Y. Chen, T. Kimura and N. Lee, Quantum Integrable Systems from Supergroup Gauge Theories, JHEP 09 (2020) 104 [arXiv:2003.13514] [INSPIRE].

[79] H.W. Braden, A. Marshakov, A. Mironov and A. Morozov, On double elliptic integrable systems. 1. A Duality argument for the case of SU(2), Nucl. Phys. B 573 (2000) 553 [hep-th/9906240] [INSPIRE].

[80] A. Mironov and A. Morozov, Commuting Hamiltonians from Seiberg-Witten theta functions, Phys. Lett. B 475 (2000) 71 [hep-th/9912088] [INSPIRE].

[81] A. Mironov and A. Morozov, Double elliptic systems: Problems and perspectives, in 3rd International Workshop on Supersymmetries and Quantum Symmetries, Moscow Russia (1999) [hep-th/0001168] [INSPIRE].

[82] G. Aminov, H.W. Braden, A. Mironov, A. Morozov and A. Zotov, Seiberg-Witten curves and double-elliptic integrable systems, JHEP 01 (2015) 033 [arXiv:1410.0698] [INSPIRE].

[83] G. Aminov, A. Mironov and A. Morozov, New non-linear equations and modular form expansion for double-elliptic Seiberg-Witten prepotential, Eur. Phys. J. C 76 (2016) 433 [arXiv: 1606.05274] [INSPIRE].

[84] G. Aminov, A. Mironov and A. Morozov, Modular properties of $6 d$ (DELL) systems, JHEP 11 (2017) 023 [arXiv: 1709.04897] [INSPIRE].

[85] M. Aganagic and A. Okounkov, Elliptic stable envelopes, J. Am. Math. Soc. 34 (2021) 79 [arXiv: 1604.00423] [INSPIRE].

[86] M. Aganagic, E. Frenkel and A. Okounkov, Quantum q-Langlands Correspondence, Trans. Moscow Math. Soc. 79 (2018) 1 [arXiv:1701.03146] [InSPIRE].

[87] M. Aganagic and A. Okounkov, Quasimap counts and Bethe eigenfunctions, Moscow Math. J. 17 (2017) 565 [arXiv: 1704.08746] [INSPIRE].

[88] A. Smirnov, Elliptic stable envelope for Hilbert scheme of points in the plane, arXiv: 1804.08779 [INSPIRE]. 